\title{
THE RESOLVENT TRACE FORMULA FOR RANK ONE LIE GROUPS*
}

\author{
YASURO GON ${ }^{\dagger}$ AND MASAO TSUZUKI ${ }^{\ddagger}$
}

\section{Introduction .}

1.1. Introduction. Let $X=G / K$ be a Riemannian symmetric space of noncompact type with $G$ a connected simple Lie group of real rank one and $K$ a maximal compact subgroup of $G$. In the paper [17], Miatello-Wallach introduced a family of bi- $K$-invariaint functions $Q_{s}, s \in \mathbf{C}$ on $G$, which satisfies the same differential equation as the elementary spherical function $\phi_{s}$ of Harish-Chandra on the open set $G^{+}=G-K$ but has singularities along $K$. By making the $r$-fold convolution of $Q_{s}$, they defined a function $Q_{r, s}$ on $G$, which is less singular than $Q_{s}$ itself. Then, given a cofinite lattice $\Gamma$ of $G$, they introduced the distribution $\mathbf{P}_{r, s}(\dot{x}, \dot{y})$ by forming the Poincaré series

$$
\mathrm{P}_{r, s}(\dot{x}, \dot{y})=c_{r}(s) \sum_{\gamma \in \Gamma} Q_{r, s}\left(x^{-1} \gamma y\right), \quad \dot{x}, \dot{y} \in \Gamma \backslash X
$$

with a suitable normalizing factor $c_{r}(s)$ and proved, among other things, that it is smooth on the complement of the diagonal in $(\Gamma \backslash X) \times(\Gamma \backslash X)$ and satisfies the differential equation

$$
\left(\triangle+\rho_{0}^{2}-s^{2}\right)^{r} \mathbf{P}_{r, s}(\dot{x},-)=\delta(\dot{x})
$$

with $\triangle$ the Laplacian of $\Gamma \backslash X, \delta(\dot{x})$ the Dirac delta supported at $\dot{x}$. In the classical situation that $X$ is the upper half plane, the distribution $\mathbf{P}_{1, s}(\dot{x}, \dot{y})$, the resolvent kernel function of Laplacian for the Riemannian surface $\Gamma \backslash X$, was intensively investigated by several German mathematicians from the view point of real analytic automorphic forms ([3], [19]). Based on these works, J. Fischer deduced the resolvent trace formula by computing the integral

$$
\int_{\Gamma \backslash X}\left(\mathbf{P}_{1, s}(\dot{x}, \dot{x})-\mathbf{P}_{1, s^{\prime}}(\dot{x}, \dot{x})\right) d \dot{x}, \quad s, s^{\prime} \in \mathbf{C}
$$

in two different ways ([5]).

In this paper, we show that the same type of procedure is possible for a higher dimensional $X$ by considering the integral $\int_{\Gamma \backslash X} \mathbf{P}_{r, s}(\dot{x}, \dot{x}) d \dot{x}$ with $r$ greater than a half of $\operatorname{dim} X$ instead of (1.3). As a result, following Fischer, we can obtain another proof of the meromorphic continuation of the Selberg zeta function for $\Gamma \backslash X$ and its functional equation, which was originally proved by Selberg, Gangolli and Gangolli-Warner ([7], $[8],[20])$.

Although a handy formula of $Q_{r, s}$ in the 'polar coordinate '(Cartan decomposition) is desirable for our purpose, it seems rather difficult to have such a formula directly from the definition of $Q_{r, s}$ recalled above. Our strategy is as follows. We first

\footnotetext{
*Received December 3, 2001; accepted for publication December 20, 2001.

†Department of Mathematics, Faculty of Science, Saitama University, 255 Shimo-ookubo, Saitama, 338-8570, Japan (ygon@rimath.saitama-u.ac.jp).

$\ddagger$ Department of Mathematics, Faculty of Science and Technology, Sophia University, Kioi-cho 7-1 Chiyoda-ku Tokyo, 102-8554, Japan (tsuzuki@mm.sophia.ac.jp).
} 
have an explicit formula of $Q_{s}=Q_{1, s}$ in terms of Gaussian hypergeometric series as in the classical case, and then use the system of differential equations among $Q_{r, s}$ 's to show that $Q_{r, s}$ is obtained from $Q_{s}$ by applying the differential operator $\left(\frac{-1}{2 s} \frac{d}{d s}\right)^{r}$ to it (Proposition 3.1.7, Theorem 3.2.1). Thus a formula of $Q_{r, s}$ in terms of a derivative of the hypergeometric series becomes available, which enables us to compute the integral $\int_{\Gamma \backslash X} \mathbf{P}_{r, s}(\dot{x}, \dot{x}) d \dot{x}$ by dividing it into the local contributions for $\Gamma$-conjugacy classes and by using various formulas involving the beta function and the hypergeometric series. Consequently we can evaluate the integral by means of the logarithmic derivative of the Selberg zeta function for $\Gamma \backslash X$. On the other hand, by the spectral expansion of $\mathbf{P}_{r, s}(\dot{x},-)$ given in [17], we compute the same integral in terms of the eigenvalues of Laplacian on $L^{2}(\Gamma \backslash X)$. Combining these two expressions of $\int_{\Gamma \backslash X} \mathbf{P}_{r, s}(\dot{x}, \dot{x}) d \dot{x}$, we arrive at the resolvent trace formula, which was studied in [5, Theorem 2.5.2, p.108] for $G=P S L_{2}(\mathbf{R})$, in [4] for $G=P S L_{2}(\mathbf{C})$ and in [1] for Jacobi forms.

Finally, we would like to say a few words on the status of our results. The resolvent trace formula (RTF for short) for a general compact locally symmetric space $\Gamma \backslash X$ with rank one $X$ is more or less known, because it is essentially the same as the determinant expression of the Selberg zeta function obtained already in [15] together with its explicit gamma factor. But we believe that our method, that is a slight extension of Fischer's, provides a more direct and elementary way to have the RTF than the traditional method employed in [20], [7] and [8], which necessitates difficult tools such as the Paley-Wiener theorem and the Plancherel formula for $X=G / K$. We also believe that our Theorem 3.2.1, that gives an expression of $Q_{r, s}$ in terms of the derivative of the hypergeometric series, is new and is interesting itself.

The authors thank Professor R. J. Miatello for his comments on the proof of Proposition 4.1.1.

2. Preliminaries. In this section we introduce basic objects and fix notations.

2.1. Notations. We denote by $\mathbf{N}$ the set of natural numbers, i.e. $\mathbf{N}=$ $\{1,2,3, \ldots\}$. Put $\mathbf{N}_{0}=\mathbf{N} \cup\{0\}$. The cardinality of a finite set $S$ is denoted by $\# S$.

Let $X$ be a set and $f, g$ two positive real valued functions defined on $X$. We write

$$
f(x) \prec g(x), \quad x \in X
$$

if there exists a positive constant $c$ such that $f(x) \leqslant c g(x)$ for all $x \in X$.

2.2. Lie groups and Lie algebras. Let $G$ be a connected semisimple Lie group of real rank one with finite center. Put $\mathfrak{g}=\operatorname{Lie}(G)$, the real Lie algebra of $G$. Let $K$ be a maximal compact subgroup of $G$ and $\theta$ the Cartan involution of $\mathfrak{g}$ corresponding to $K$, then we have the Cartan decomposition $\mathfrak{g}=\mathfrak{k}+\mathfrak{p}$ with $\mathfrak{k}=\operatorname{Lie}(K)$. We fix an Iwasawa decomposition $G=N A K$ of $G ; A$ is a maximal split torus in $G$ whose Lie algebra $\mathfrak{a}$ is orthogonal to $\mathfrak{k}$ with respect to the Killing form $B$ of $G$ and $N$ a maximal unipotent subgroup of $G$ normalized by $A$. Since $\operatorname{dim} A=1$ by assumption, there exists a unique root $\alpha \in \mathfrak{a}^{*} \operatorname{such}$ that $\mathfrak{n}_{j \alpha}=\{X \in \mathfrak{g} \mid \operatorname{ad}(H) X=j \cdot \alpha(H) X, H \in \mathfrak{a}\}$ with $j \in \mathbf{Z}$ is zero if $|j|>2$, and $\operatorname{Lie}(N)=\mathfrak{n}=\mathfrak{n}_{\alpha}+\mathfrak{n}_{2 \alpha}$.

Let $H_{0}$ be the unique element of $\mathfrak{a}$ such that $\alpha\left(H_{0}\right)=1$. Let $\langle\rangle:, \mathfrak{a} \times \mathfrak{a} \rightarrow \mathbf{R}$ be the inner product induced by $B$; it gives the identification $\mathfrak{a} \cong \mathfrak{a}^{*}$. The dual inner product of $\mathfrak{a}^{*}$ is also denoted by $\langle$,$\rangle . Put p=\operatorname{dim}_{\mathbf{R}} \mathfrak{n}_{\alpha}, q=\operatorname{dim}_{\mathbf{R}} \mathfrak{n}_{2 \alpha}, \rho_{0}=2^{-1}(p+2 q)$, $c_{0}=(2 p+8 q)^{-1}$ and $m=2^{-1} \operatorname{dim}(G / K)$. Then by the classification, we have the list: 


\begin{tabular}{c|c|c|c|c|}
$\mathfrak{g}$ & $p$ & $q$ & $\rho_{0}$ & $m$ \\
\hline $\mathfrak{s u}(l, 1)$ & $2 l-2$ & 1 & $l$ & $l$ \\
$\mathfrak{s o}(l, 1)$ & $l-1$ & 0 & $2^{-1}(l-1)$ & $2^{-1} l$ \\
$\mathfrak{s p}(l, 1)$ & $4 l-4$ & 1 & $2 l-1$ & $2 l-1$ \\
$\mathfrak{f}_{4(-20)}$ & 8 & 7 & 11 & 8
\end{tabular}

( $l$ is a natural number greater than one.)

LEMMA 2.2.1. We have

$$
2 m=p+q+1, \quad\left\langle H_{0}, H_{0}\right\rangle=c_{0}^{-1}, \quad\langle\alpha, \alpha\rangle=c_{0} .
$$

From now on we assume that $m \in \mathbf{N}, m \geqslant 2$. In other words, we exclude the case of $\mathfrak{g} \cong \mathfrak{s l}_{2}(\mathbf{R})$ or $\mathfrak{s o}(2 l+1,1)$ with $l \geqslant 1$.

2.3. Haar measures. Let $d k$ be the Haar measure of the compact group $K$ with total mass one. Let $d t$ be the standard Lebesgue measure of $\mathbf{R}$; by the identification $\mathbf{R} \cong A=\exp \mathfrak{a}, t \mapsto \exp \left(t H_{0}\right)$, it gives the Haar measure of the torus $A$. Denote by $C_{\mathrm{c}}^{0}(N)$ the space of compactly supported continuous functions on $N$. Since $N=$ $\exp \left(\mathfrak{n}_{\alpha}+\mathfrak{n}_{2 \alpha}\right)$ is a unipotent Lie group we can take its Haar measure $d n$ such that the formula

$$
\int_{N} f(n) d n=\int_{\mathfrak{n}_{\alpha}} \int_{\mathfrak{n}_{2 \alpha}} f(\exp (X+Y)) d X d Y, \quad f \in C_{\mathrm{c}}^{0}(N)
$$

holds with $d X$ (resp. $d Y$ ) the Euclidian measure of $\mathfrak{n}_{\alpha}\left(\right.$ resp. $\left.\mathfrak{n}_{2 \alpha}\right)$. (We regard $\mathfrak{n}_{j \alpha}$ as a Euclidean space by the inner product $-B(Z, \theta Z)$.)

Then we fix the Haar measure $d g$ of $G=A N K$ by $d g=d a \cdot d n \cdot d k$. To handle various bi- $K$-invariant functions (distributions) on $G$, the Cartan decomposition $G=$ $K \exp \left([0, \infty) H_{0}\right) K$ is indispensable. We put

$$
G^{+}=G-K=K A^{+} K \text { with } A^{+}=\left\{\exp \left(t H_{0}\right) \mid t>0\right\} .
$$

If $g \in G^{+}$, and $g=k_{1}(g) a(g) k_{2}(g)$, with $k_{1}(g), k_{2}(g) \in K$ and $a(g) \in A^{+}$, then $a(g)$ is uniquely determined by $g$. We choose the Riemannian metric $d x$ on $X=G / K$, induced by the restriction $\left.B\right|_{\mathfrak{p}}$ of $B$ to $\mathfrak{p}$. We then have that the hyperbolic distance $d(x K, y K)=B\left(t H_{0}, t H_{0}\right)^{1 / 2}=t$ if $x, y \in G$ and $a\left(x^{-1} y\right)=\exp \left(t H_{0}\right)$, with $t>0$.

The measure $d g$ on $G$ is decomposed along the Cartan decomposition as follows.

LEMma 2.3.1. For any positive measurable function $\varphi$ on $G$, the formula

$$
\int_{G} \varphi(g) d g=c_{G} \int_{K} \int_{0}^{\infty} \int_{K} \varphi\left(k_{1} \exp \left(t H_{0}\right) k_{2}\right) \mu(t) d k_{1} d t d k_{2}
$$

holds. Here

$$
\begin{aligned}
& \mu(t)=(\sinh t)^{p+q}(\cosh t)^{q}, \\
& c_{G}=2 \Gamma(m)^{-1}\left(2^{-1} c_{0}\right)^{-m+1 / 2} \pi^{m} .
\end{aligned}
$$

Proof. Define the function $f$ on $G$ by

$$
f\left(k_{1} \exp \left(t H_{0}\right) k_{2}\right)=(\cosh t)^{2 s} \quad\left(k_{1}, k_{2} \in K, t \in \mathbf{R}\right) .
$$

Next integrate $f$ in the both hand sides of (2.1). 
3. Spherical functions. In the first subsection, after recalling the standard properties of zonal spherical functions for $G / K$, we introduce a bi- $K$-invariant function $\phi_{s}^{(2)}$ on $G^{+}$with singularities along $K$, which is called the secondary spherical function by T. Oda ([18]). We investigate its properties in some detail to show that its $r$-times derivative with respect to $s^{2}$ gives the function $Q_{r, s}$ of Miatello-Wallach ([17]).

3.1. The spherical function with singularities. For $s \in \mathbf{C}$, the zonal spherical function $\phi_{s}$ for $G / K$ is defined by the integral

$$
\phi_{s}(g)=\int_{K} e^{\left(s+\rho_{0}\right) \alpha(H(k g))} d k, \quad g \in G .
$$

Here for $g \in G, H(g)$ denotes the unique vector in a such that $g \in N \exp (H(g)) K$. The basic property of $\phi_{s}$ is listed below.

(a) It is bi- $K$-invariant $C^{\infty}$-function on $G$, i.e., $\phi_{s} \in C^{\infty}(K \backslash G / K)$.

(b) It satisfies the differential equation

$$
\Omega \phi_{s}(g)=\left(s^{2}-\rho_{0}^{2}\right) \phi_{s}(g), \quad g \in G
$$

with $\Omega$ the Casimir element of $G$ corresponding to $c_{0} B$.

(c) If $\operatorname{Re}(s)>0$, then

$$
\lim _{t \rightarrow+\infty} e^{t\left(\rho_{0}-s\right)} \phi_{s}\left(\exp \left(t H_{0}\right)\right)=c(s)
$$

with $c(s)$ the $c$-function for $G / K$ given by

$$
c(s)=2^{\rho_{0}-s} \Gamma(m) \Gamma(s) \Gamma\left(\frac{s+\rho_{0}}{2}\right)^{-1} \Gamma\left(\frac{s-\rho_{0}+2 m}{2}\right)^{-1} .
$$

Put $u_{s}^{(1)}(t)=\phi_{s}\left(\exp \left(t H_{0}\right)\right), t \in \mathbf{R}$. Then by (a), $u_{s}^{(1)}(t)$ is a $C^{\infty}$-function on $\mathbf{R}$ which determines $\phi_{s}$ uniquely, and by (b) it satisfies the ordinary second order differential equation

$$
(D)_{s}: \quad \frac{d^{2} u}{d t^{2}}+\left(\frac{p}{\tanh t}+\frac{q}{\tanh (2 t)}\right) \frac{d u}{d t}+\left(\rho_{0}^{2}-s^{2}\right) u=0
$$

which has the regular singularity at $t=0$ with characteristic exponents $\{0,2-$ $2 m\}$. Change the variable by $z=\tanh ^{2} t$ and consider the function $w(z)=$ $(\cosh t)^{\rho_{0}-s} u_{s}^{(1)}(t)$. Then it turns out that $w$ is a solution of the Gaussian hypergeometric differential equation $z(1-z) \frac{d^{2} w}{d z^{2}}+\{c-(1+a+b) z\} \frac{d w}{d z}-a b w=0$ with $a=2^{-1}\left(-s+\rho_{0}\right), b=2^{-1}\left(-s+\rho_{0}-q+1\right)$ and $c=m$. Thus we have

$$
u_{s}^{(1)}(t)=(\cosh t)^{s-\rho_{0}} F_{1}\left(\frac{-s+\rho_{0}}{2}, \frac{-s+\rho_{0}-q+1}{2} ; m ; \tanh ^{2} t\right), \quad t \in \mathbf{R} .
$$

We are interested in another class of solutions of $(D)_{s}$ which admit a singularity at $t=0$. Among them, the one with the fastest decay at infinity, which we now define, is of particular importance: For $s \in \mathbf{C}-\{-1,-2,-3, \ldots\}$, put

$$
\begin{aligned}
& u_{s}^{(2)}(t)=\gamma(s)(\cosh t)^{-\left(s+\rho_{0}\right)}{ }_{2} F_{1}\left(\frac{s+\rho_{0}}{2}, \frac{s-\rho_{0}+2 m}{2} ; s+1 ; \frac{1}{\cosh ^{2} t}\right), t \in \mathbf{R}-\{0\}, \\
& \gamma(s)=\Gamma\left(\frac{s+\rho_{0}}{2}\right) \Gamma\left(\frac{s-\rho_{0}+2 m}{2}\right) \Gamma(s+1)^{-1} \Gamma(m-1)^{-1}=2^{-s+\rho_{0}} m(s c(s))^{-1} .
\end{aligned}
$$

Proposition 3.1.1. 
(i) If $s \in \mathbf{C}$ is not a pole of $\gamma(s)$ and $\gamma(s) \neq 0$, then the family $\left\{u_{s}^{(1)}, u_{s}^{(2)}\right\}$ gives a system of fundamental solutions of $(D)_{s}$ around $t=0$.

(ii) There exists a unique family of functions $\left\{\phi_{s}^{(2)} \mid \operatorname{Re}(s) \geqslant 0\right\}$ in $C^{\infty}\left(K \backslash G^{+} / K\right)$ such that

(a)

$$
\Omega \phi_{s}^{(2)}(g)=\left(s^{2}-\rho_{0}^{2}\right) \phi_{s}^{(2)}(g), \quad g \in G^{+}
$$

(b)

$$
\phi_{s}^{(2)}\left(\exp \left(t H_{0}\right)\right)=O\left(e^{-t\left(\operatorname{Re}(s)+\rho_{0}\right)}\right), \quad(t \rightarrow+\infty),
$$

(c)

$$
\lim _{t \rightarrow+0} t^{2 m-2} \phi_{s}^{(2)}\left(\exp \left(t H_{0}\right)\right)=1
$$

For a given $g \in G^{+}$the function $s \mapsto \phi_{s}^{(2)}(g)$ is holomorphic on $\operatorname{Re}(s) \geqslant 0$. We have $\phi_{s}^{(2)}\left(\exp \left(t H_{0}\right)\right)=u_{s}^{(2)}(t)$ for $t \in \mathbf{R}-\{0\}$.

Proof. (i) Let us consider the Wronskian of the $\left\{u_{s}^{(1)}, u_{s}^{(2)}\right\}$, put

$$
W\left[u_{s}^{(1)}, u_{s}^{(2)}\right](t)=u_{s}^{(1)}(t) \cdot \frac{d}{d t} u_{s}^{(2)}(t)-\frac{d}{d t} u_{s}^{(1)}(t) \cdot u_{s}^{(2)}(t) .
$$

It is easy to verify that $\lim _{t \rightarrow+0} u_{s}^{(1)}(t)=1, \lim _{t \rightarrow+0} \frac{d}{d t} u_{s}^{(1)}(t)=0$, $\lim _{t \rightarrow+0} t^{2 m-2} u_{s}^{(2)}(t)=1$ and $\lim _{t \rightarrow+0} t^{2 m-1} \frac{d}{d t} u_{s}^{(2)}(t)=2-2 m$. So we have

$$
\begin{aligned}
& \lim _{t \rightarrow+0} t^{2 m-1} W\left[u_{s}^{(1)}, u_{s}^{(2)}\right](t) \\
= & \lim _{t \rightarrow+0}\left\{u_{s}^{(1)}(t) \cdot t^{2 m-1} \frac{d}{d t} u_{s}^{(2)}(t)-t \frac{d}{d t} u_{s}^{(1)}(t) \cdot t^{2 m-2} u_{s}^{(2)}(t)\right\} \\
= & 2-2 m \neq 0 .
\end{aligned}
$$

Hence there exists a $t_{0}, 0<t_{0}<\epsilon$ with a small $\epsilon>0$, such that $W\left[u_{s}^{(1)}, u_{s}^{(2)}\right]\left(t_{0}\right) \neq 0$. This means that the Wronskian $W\left[u_{s}^{(1)}, u_{s}^{(2)}\right](t)$ does not vanish identically and we have the assertion .

(ii) By the condition (a) and the fact (i), $\phi_{s}^{(2)}$ can be written as a linear combination of $u_{s}^{(1)}$ and $u_{s}^{(2)}$ as

$$
\phi_{s}^{(2)}\left(\exp \left(t H_{0}\right)\right)=\alpha_{1} \cdot u_{s}^{(1)}(t)+\alpha_{2} \cdot u_{s}^{(2)}(t) \quad(t>0) .
$$

Since $\lim _{t \rightarrow+0} t^{2 m-2} u_{s}^{(1)}(t)=0$ and $\lim _{t \rightarrow+0} t^{2 m-2} u_{s}^{(2)}(t)=1$, the condition (c) implies that $\alpha_{2}=1$. Let us check $\alpha_{1}=0$. Firstly we assume that $\operatorname{Re}(s)>$ 0 . By the explicit formula, we have $\lim _{t \rightarrow+\infty} e^{\left(\rho_{0}-s\right) t} u_{s}^{(1)}(t)=c(s) \neq 0$ and $\lim _{t \rightarrow+\infty} e^{\left(\rho_{0}-s\right) t} u_{s}^{(2)}(t)=0$. So we obtain $\alpha_{1}=0$ by the condition (b). Secondly we assume that $\operatorname{Re}(s)=0$. Then we have $u_{s}^{(1)}(t)=O\left(\log (\cosh t) e^{-\rho_{0} t}\right)$ and $u_{s}^{(2)}(t)=O\left(e^{-\rho_{0} t}\right)$ when $t$ tends to $+\infty$. Thus we obtain $\alpha_{1}=0$ by (b).

Proposition 3.1.2. Put

$$
=\frac{(-1)^{m} \pi}{\Gamma(m) \Gamma(m-1)} \cdot \prod_{j=1}^{m-1}\left\{\left(\frac{s}{2}\right)^{2}-\left(\frac{\rho_{0}}{2}-j\right)^{2}\right\} \cdot\left\{\cot \left(\frac{s-\rho_{0}}{2} \pi\right)+\cot \left(\frac{s+\rho_{0}}{2} \pi\right)\right\} \text {. }
$$


Then for $s \in \mathbf{C}$ with $|\operatorname{Re}(s)|<1$, we have

$$
\phi_{-s}^{(2)}(g)=\phi_{s}^{(2)}(g)+c(s) \phi_{s}(g), \quad g \in G^{+} .
$$

Proof. The function $u_{-s}^{(2)}(t)$ on $t>0$ is a solution of $(D)_{s}$. Hence by Proposition 3.1 .1 , it can be written as a linear combination of $u_{s}^{(1)}$ and $u_{s}^{(2)}$ as

$$
u_{-s}^{(2)}(t)=c_{1} \cdot u_{s}^{(1)}(t)+c_{2} \cdot u_{s}^{(2)}(t) \quad(t>0)
$$

Since $\lim _{t \rightarrow+0} t^{2 m-2} u_{s}^{(1)}(t)=0$ and $\lim _{t \rightarrow+0} t^{2 m-2} u_{s}^{(2)}(t)=\lim _{t \rightarrow+0} t^{2 m-2} u_{-s}^{(2)}(t)=1$, we have $c_{2}=1$.

Let us determine $c_{1}$. First we assume that $0<\operatorname{Re}(s)<1$. By the explicit formula, we have $\lim _{t \rightarrow+\infty} e^{\left(\rho_{0}-s\right) t} u_{s}^{(2)}(t)=0$ and $\lim _{t \rightarrow+\infty} e^{\left(\rho_{0}-s\right) t} u_{-s}^{(2)}(t)=\gamma(-s) \cdot 2^{\rho_{0}-s}$. So we obtain

$$
2^{\rho_{0}-s} \gamma(-s)=c_{1} \cdot 2^{\rho_{0}-s} \Gamma(s) \Gamma(m) \Gamma\left(\frac{s+\rho_{0}}{2}\right)^{-1} \Gamma\left(\frac{s-\rho_{0}+2 m}{2}\right)^{-1}
$$

Thus

$$
\begin{aligned}
c_{1}= & (\Gamma(m) \Gamma(m-1) \Gamma(s) \Gamma(1-s))^{-1} \\
& \times \Gamma\left(\frac{-s+\rho_{0}}{2}\right) \Gamma\left(\frac{s+\rho_{0}}{2}\right) \Gamma\left(\frac{-s-\rho_{0}+2 m}{2}\right) \Gamma\left(\frac{s-\rho_{0}+2 m}{2}\right) .
\end{aligned}
$$

Note that

$$
\begin{aligned}
\Gamma(s) \Gamma(1-s) & =\pi \sin (\pi s)^{-1} \\
\Gamma\left(\frac{s-\rho_{0}+2 m}{2}\right) & =\Gamma\left(m-\frac{-s+\rho_{0}}{2}\right) \\
& =\prod_{j=1}^{m-1}\left(m-j-\frac{-s+\rho_{0}}{2}\right) \cdot \Gamma\left(1-\frac{-s+\rho_{0}}{2}\right) \\
\Gamma\left(\frac{s+\rho_{0}}{2}\right)=\Gamma\left(m-\frac{-s-\rho_{0}+2 m}{2}\right) & \\
= & \prod_{j=1}^{m-1}\left(m-j-\frac{-s-\rho_{0}+2 m}{2}\right) \cdot \Gamma\left(1-\frac{-s-\rho_{0}+2 m}{2}\right)
\end{aligned}
$$

Then we have

$$
\begin{aligned}
c_{1}= & \frac{1}{\Gamma(m) \Gamma(m-1)} \frac{\sin (\pi s)}{\pi} \\
& \times \prod_{j=1}^{m-1}\left\{\left(m-j-\frac{-s+\rho_{0}}{2}\right)\left(m-j-\frac{-s-\rho_{0}+2 m}{2}\right)\right\} \\
& \times \Gamma\left(\frac{-s+\rho_{0}}{2}\right) \Gamma\left(1-\frac{-s+\rho_{0}}{2}\right) \Gamma\left(\frac{-s-\rho_{0}+2 m}{2}\right) \Gamma\left(1-\frac{-s-\rho_{0}+2 m}{2}\right) \\
= & \frac{1}{\Gamma(m) \Gamma(m-1)} \frac{\sin (\pi s)}{\pi} \prod_{j=1}^{m-1}\left\{\left(\frac{s}{2}\right)^{2}-\left(\frac{\rho_{0}}{2}-j\right)^{2}\right\} \\
& \times \frac{\pi}{\sin \left(\frac{-s+\rho_{0}}{2} \pi\right)} \frac{\pi}{\sin \left(\frac{-s-\rho_{0}+2 m}{2} \pi\right)} .
\end{aligned}
$$


Since

$\frac{\sin (\pi s)}{\sin \left(\frac{-s+\rho_{0}}{2} \pi\right) \sin \left(\frac{-s-\rho_{0}+2 m}{2} \pi\right)}=(-1)^{m-1}\left\{\cot \left(\frac{-s+\rho_{0}}{2} \pi\right)+\cot \left(\frac{-s-\rho_{0}+2 m}{2} \pi\right)\right\}$

the formula (3.2) is now established for $0<\operatorname{Re}(s)<1$. By the analytic continuation, the formula remains valid on $|\operatorname{Re}(s)|<1$.

The 'bad 'behavior of the function $\phi_{s}^{(2)}\left(\exp \left(t H_{0}\right)\right)$ near $t=0$ is controlled by a simple function. Indeed, we have

Proposition 3.1.3. There exists a function $(s, t) \mapsto \mathrm{Y}_{s}(t)$ on $\mathbf{C} \times(\mathbf{R}-\{0\})$ with the following properties

(a) We can write

$$
\begin{aligned}
\mathrm{Y}_{s}(t)= & \sum_{j=0}^{m-1} \frac{\mathrm{a}_{j}(s)}{(\sinh t)^{2 j}}+\mathrm{b}(s) \log \left(\sinh ^{2} t\right) \\
& +\frac{\mathrm{b}(s)}{\Gamma(m) \Gamma(m-1)}\left\{\psi\left(\frac{s+\rho_{0}}{2}\right)+\psi\left(\frac{s-\rho_{0}}{2}+1\right)\right\}
\end{aligned}
$$

with polynomial functions $\mathrm{a}_{j}(s)$ and $\mathrm{b}(s)$ such that

$$
\begin{aligned}
& \mathrm{a}_{j}(-s)=\mathrm{a}_{j}(s), \quad \operatorname{deg}\left(\mathrm{a}_{j}(s)\right) \leqslant 2(m-j-1), \quad j=0, \ldots, m-1, \\
& \mathrm{a}_{m-1}(s)=1, \\
& \mathrm{~b}(s)=(-1)^{m} \prod_{j=1}^{m-1}\left\{\left(\frac{s}{2}\right)^{2}-\left(\frac{\rho_{0}}{2}-j\right)^{2}\right\} .
\end{aligned}
$$

Here $\psi(s)$ is the digamma function, i.e., the logarithmic derivative of the Gamma function.

(b) There exists a family of polynomial functions $\left\{\mathrm{c}_{n}(s)\right\}_{n \geqslant 1}$ and $\left\{\mathrm{d}_{n}(s)\right\}_{n \geqslant 1}$ such that $\sum_{n=1}^{\infty} \mathrm{c}_{n}(s) t^{n}$ and $\sum_{n=1}^{\infty} \mathrm{d}_{n}(s) t^{n}$ have positive radius of convergence and such that

$$
\phi_{s}^{(2)}\left(\exp \left(t H_{0}\right)\right)=\mathrm{Y}_{s}(t)+\sum_{n=1}^{\infty} c_{n}(s) t^{n}+\log (t) \sum_{n=1}^{\infty} \mathrm{d}_{n}(s) t^{n}
$$

on $0<t<\epsilon$ with a small $\epsilon>0$.

Proof. Put $u=\sinh ^{2} t, a=2^{-1}\left(s-\rho_{0}\right)+1$ and $b=2^{-1}\left(s+\rho_{0}-2 m\right)+1$. Then

$$
u_{s}^{(2)}(t)=\gamma(s)(1+u)^{\left(2 m-\rho_{0}-s\right) / 2-1}{ }_{2} F_{1}\left(a, b ; a+b+m-1 ; \frac{1}{1+u}\right) .
$$


By the formula $[16,(9.7 .5), \mathrm{p} .257]$, we have

$$
\begin{aligned}
u_{s}^{(2)}(t)= & \gamma(s) \cdot(1+u)^{\frac{2 m-\rho_{0}-s}{2}-1} u^{1-m} \\
& \times\left\{\frac{\Gamma(a+b+m-1)}{\Gamma(a+m-1) \Gamma(b+m-1)} \sum_{k=0}^{m-2} \frac{(-1)^{k}(m-k-2) !}{k !}(a)_{k}(b)_{k}\left(\frac{u}{1+u}\right)^{k}\right. \\
& +\frac{(-1)^{m-1} \Gamma(a+b+m-1)}{\Gamma(a) \Gamma(b)} \sum_{k=0}^{\infty} \frac{(a+m-1)_{k}(b+m-1)_{k}}{(m+k-1) ! k !} \\
& \times(\psi(k+1)+\psi(m+k)-\psi(a+m+k-1)-\psi(b+m+k-1) \\
= & \left.\left.\quad-\log \left(\frac{u}{1+u}\right)\right) \cdot\left(\frac{u}{1+u}\right)^{k+m-1}\right\} \\
& +(-1)^{m-1} \sum_{k=0}^{m-2} \frac{(-1)^{k}(m-k-2) !}{(m-2) ! k !}(a)_{k}(b)_{k} u^{k-m+1}(1+u)^{\frac{2 m-\rho_{0}-s}{2}-1-k} \\
& \times \begin{cases}(m+1)+(m-2) !(m+k-1) ! \\
\end{cases} \\
& \left.\quad-\log \left(\frac{u}{1+u}\right)\right\} u^{k}(1+u)^{-\frac{\rho_{0}+s}{2}-k} \quad(0<|u|<\epsilon)
\end{aligned}
$$

with a small $\epsilon$. put

Note that $\gamma(s)=\Gamma(a+m-1) \Gamma(b+m-1) \Gamma(a+b+m-1)^{-1} \Gamma(m-1)^{-1}$. Now

$$
\begin{aligned}
A_{k}(s)= & \frac{(-1)^{k}(m-k-2) !}{k !(m-2) !}(a)_{k}(b)_{k} \quad(k=0, \ldots, m-2) \\
B_{k}(s)= & \frac{(a+m-1)_{k}(b+m-1)_{k}(a)_{m-1}(b)_{m-1}}{k !(m-2) !(m+k-1) !} \quad\left(k \in \mathbf{N}_{0}\right) \\
\gamma_{k}= & \psi(k+1)+\psi(m+k) \\
& -\psi(a+m+k-1)-\psi(b+m+k-1) \quad\left(k \in \mathbf{N}_{0}\right)
\end{aligned}
$$

and

$$
\begin{aligned}
& (1+u)^{-b-k}=\sum_{n=0}^{\infty} c_{n, k}^{+}(s) u^{n} \quad(|u|<1), \\
& (1+u)^{-\frac{s+\rho_{0}}{2}-k}=\sum_{n=0}^{\infty} c_{n, k}^{-}(s) u^{n} \quad(|u|<1),
\end{aligned}
$$

with $\quad c_{n, k}^{+}(s)=\frac{(-1)^{n}}{n !}\left(\frac{s+\rho_{0}}{2}-m+k+1\right)_{n}, \quad c_{n, k}^{-}(s)=\frac{(-1)^{n}}{n !}\left(\frac{s+\rho_{0}}{2}+k\right)_{n}$. 
Then

$$
\begin{aligned}
u_{s}^{(2)}(t)= & \sum_{n=0}^{\infty} \sum_{k=0}^{m-2} A_{k}(s) c_{n, k}^{+}(s) u^{k+n-m+1} \\
& +(-1)^{m-1} \sum_{k=0}^{\infty} \sum_{n=0}^{\infty} B_{k}(s) c_{n, k}^{-}(s)\left\{\gamma_{k}-\log \left(\frac{u}{1+u}\right)\right\} u^{k+n} \\
= & \sum_{k=1}^{m-1} \frac{a_{k}(s)}{u^{k}}+\mathrm{b}(s) \log (u)+\tilde{a_{0}}(s) \\
& +\left\{\sum_{k=1}^{\infty} p_{k}(s) u^{k}+\log (u) \sum_{k=1}^{\infty} q_{k}(s) u^{k}\right\}
\end{aligned}
$$

with $\mathrm{b}(s)=(-1)^{m} B_{0}(s)$,

$$
\begin{aligned}
& \mathrm{a}_{j}(s)=\sum_{\substack{n \geqslant 0,0 \leqslant k<m-1 \\
k+n-m+1=-j}} A_{k}(s) c_{n, k}^{+}(s) \quad(j=1, \ldots, m-1), \\
& \tilde{a_{0}}(s)=\sum_{k=0}^{m-2} A_{k}(s) c_{m-1-k, k}^{+}(s)+(-1)^{m-1} B_{0}(s) \gamma_{0}
\end{aligned}
$$

and $p_{k}(s), q_{k}(s)$ are some polynomials. By the foumulae of $A_{k}$ and $c_{n, k}^{+}$, we see that $A_{k}(s) c_{n, k}^{+}(s)$ with $n \geqslant 0,0 \leqslant k<m-1, k+n-m+1=-j$ is a polynomial of $s$ such that $\operatorname{deg}\left(A_{k}(s) c_{n, k}^{+}(s)\right)=2 k+n=k-j+m-1$. Hence $\mathrm{a}_{j}(s)$ is also a polynomial with

$$
\operatorname{deg}\left(a_{j}(s)\right) \leqslant \max _{\substack{n \geqslant 0,0 \leqslant k<m-1 \\ k+n-m+1=-j}}\{k-j+m-1\}=2 m-2 j-2=2(m-j-1)
$$

We have

$$
\begin{aligned}
B_{0}(s) & =\frac{(a)_{m-1}(b)_{m-1}}{(m-2) !(m-1) !} \\
& =\frac{1}{\Gamma(m-1) \Gamma(m)}\left(\frac{s-\rho_{0}}{2}+1\right)_{m-1}\left(\frac{s+\rho_{0}-2 m}{2}+1\right)_{m-1} \\
& =\frac{1}{\Gamma(m-1) \Gamma(m)} \prod_{j=0}^{m-2}\left(\frac{s-\rho_{0}}{2}+j+1\right)\left(\frac{s+\rho_{0}-2 m}{2}+j+1\right) \\
& =\frac{1}{\Gamma(m-1) \Gamma(m)} \prod_{j=1}^{m-1}\left(\frac{s-\rho_{0}}{2}+j\right)\left(\frac{s+\rho_{0}}{2}-m+j\right) \\
& =\frac{1}{\Gamma(m-1) \Gamma(m)} \prod_{j=1}^{m-1}\left\{\left(\frac{s}{2}\right)^{2}-\left(\frac{\rho_{0}}{2}-j\right)^{2}\right\}
\end{aligned}
$$




$$
\begin{aligned}
\tilde{a_{0}}(s)= & \frac{(-1)^{m}}{\Gamma(m-1) \Gamma(m)} \prod_{j=1}^{m-1}\left\{\left(\frac{s}{2}\right)^{2}-\left(\frac{\rho_{0}}{2}-j\right)^{2}\right\} \\
& \times\left\{\psi\left(\frac{s-\rho_{0}}{2}+m\right)+\psi\left(\frac{s+\rho_{0}}{2}\right)-\psi(1)-\psi(m)\right\} \\
& +\sum_{k=0}^{m-2} A_{k}(s) c_{m-1-k, k}^{+}(s) .
\end{aligned}
$$

Using the formula

$$
\psi(y+m)=\psi(y+1)+\sum_{j=1}^{m-1} \frac{1}{y+m-j} \quad\left(m \in \mathbf{N}_{0}\right),
$$

we have

$$
\begin{aligned}
& \tilde{\mathrm{a}_{0}(s)=} \frac{(-1)^{m}}{\Gamma(m-1) \Gamma(m)} \prod_{j=1}^{m-1}\left\{\left(\frac{s}{2}\right)^{2}-\left(\frac{\rho_{0}}{2}-j\right)^{2}\right\} \cdot\left\{\psi\left(\frac{s-\rho_{0}}{2}+1\right)+\psi\left(\frac{s+\rho_{0}}{2}\right)\right\} \\
& \quad+\mathrm{a}_{0}(s) \\
& \text { with } \mathrm{a}_{0}(s)=\prod_{j=1}^{m-1}\left\{\left(\frac{s}{2}\right)^{2}-\left(\frac{\rho_{0}}{2}-j\right)^{2}\right\} \cdot\left(\sum_{j=1}^{m-1} \frac{2}{s-\rho_{0}+2 m-2 j}-\psi(1)-\psi(m)\right) \\
& \quad+\sum_{k=0}^{m-2} A_{k}(s) c_{m-1-k, k}^{+}(s) .
\end{aligned}
$$

By the formulae $A_{k}(s)$ and $c_{n, k}^{+}(s)$, we have that $\mathrm{a}_{0}(s)$ is a polynomial function with degree no more than $2(m-1)$. Put

$$
\begin{aligned}
\mathrm{Y}_{s}(t):= & \sum_{j=0}^{m-1} \frac{\mathrm{a}_{j}(s)}{(\sinh t)^{2 j}}+\mathrm{b}(s) \log \left(\sinh ^{2} t\right) \\
& \quad+\frac{\mathrm{b}(s)}{\Gamma(m) \Gamma(m-1)}\left\{\psi\left(\frac{s+\rho_{0}}{2}\right)+\psi\left(\frac{s-\rho_{0}}{2}+1\right)\right\} .
\end{aligned}
$$

Then all the assertions in Proposition 3.1.3, except that $\mathrm{a}_{j}(s)$ 's are even functions are established. To have $a_{j}(s)=a_{j}(-s)$, we use the functional equation in Proposition 3.1.2. From that equation

$$
\begin{aligned}
& a_{j}(-s)=a_{j}(s) \quad(j=1, \ldots, m-1), \\
& \tilde{a_{0}}(-s)=\tilde{a_{0}}(s)+c(s) .
\end{aligned}
$$

From (3.17) and (3.21), we have

$$
\begin{aligned}
& \mathrm{a}_{0}(-s)-\mathrm{a}_{0}(s) \\
& =\mathrm{c}(s)+\frac{(-1)^{m}}{\Gamma(m-1) \Gamma(m)} \prod_{j=1}^{m-1}\left\{\left(\frac{s}{2}\right)^{2}-\left(\frac{\rho_{0}}{2}-j\right)^{2}\right\} \\
& \quad \times\left\{\psi\left(\frac{s-\rho_{0}}{2}+1\right)+\psi\left(\frac{s+\rho_{0}}{2}\right)-\psi\left(\frac{-s-\rho_{0}}{2}+1\right)-\psi\left(\frac{-s+\rho_{0}}{2}\right)\right\} \\
& =\frac{(-1)^{m}}{\Gamma(m-1) \Gamma(m)} \prod_{j=1}^{m-1}\left\{\left(\frac{s}{2}\right)^{2}-\left(\frac{\rho_{0}}{2}-j\right)^{2}\right\} \cdot \Delta(s)
\end{aligned}
$$


with

$$
\begin{aligned}
\triangle(s)= & \pi \cot \left(\frac{s-\rho_{0}}{2} \pi\right)+\pi \cot \left(\frac{s+\rho_{0}-2 m}{2} \pi\right)+\psi\left(\frac{s-\rho_{0}}{2}+1\right) \\
& +\psi\left(\frac{s+\rho_{0}}{2}\right)-\psi\left(\frac{-s-\rho_{0}}{2}+1\right)-\psi\left(\frac{-s+\rho_{0}}{2}\right) .
\end{aligned}
$$

We claim $\triangle(s) \equiv 0$. Indeed, the formula $\psi(y)-\psi(1-y)=-\pi \cot (\pi y)$ gives

$$
\begin{aligned}
-\psi\left(\frac{-s+\rho_{0}}{2}\right)+\psi\left(1-\frac{-s+\rho_{0}}{2}\right)+\pi \cot \left(\frac{s-\rho_{0}}{2} \pi\right) & =0 \\
\psi\left(\frac{s+\rho_{0}}{2}\right)-\psi\left(1-\frac{s+\rho_{0}}{2}\right)+\pi \cot \left(\frac{s+\rho_{0}}{2} \pi\right) & =0 .
\end{aligned}
$$

Since $\pi \cot (\pi y+m \pi)=\pi \cot (\pi y)$, we have $\triangle(s)=0$.

We introduce a family of functions $\phi_{s}^{[r]}\left(\operatorname{Re}(s)>-1, r \in \mathbf{N}_{0}\right)$ as

Definition 3.1.4. For $r \in \mathbf{N}_{0}$, we put

$$
\phi_{s}^{[r]}(g)=\frac{c_{G}^{-1}}{(2-2 m) r !}\left(-\frac{1}{2 s} \frac{d}{d s}\right)^{r} \phi_{s}^{(2)}(g), \quad g \in G^{+}, \quad \operatorname{Re}(s)>-1 .
$$

The basic property of $\phi_{s}^{[r]}$ we need is as follows.

Proposition 3.1.5. Let $r \in \mathbf{N}_{0}$ and $s \in \mathbf{C}$ with $\operatorname{Re}(s)>0$.

(i) The function $\phi_{s}^{[r]}$ belongs to $C^{\infty}\left(K \backslash G^{+} / K\right)$.

(ii) We have

$$
\phi_{s}^{[r]}\left(\exp \left(t H_{0}\right)\right)=O\left(e^{-t\left(\operatorname{Re}(s)+\rho_{0}\right)}\right)
$$

on $t>R$ with a large $R>0$.

(iii) If $r \geqslant m$, then the function $\phi_{s}^{[r]}$ has a continuous extension to all of $G$. We have

$$
\begin{aligned}
& \lim _{g \rightarrow e, g \in G^{+}} \phi_{s}^{[r]}(g) \\
= & \frac{-c_{G}^{-1}}{2 r !}\left(-\frac{1}{2 s} \frac{d}{d s}\right)^{r}\left[\frac{\mathrm{b}(s)}{\Gamma(m)^{2}}\left\{\psi\left(\frac{s+\rho_{0}}{2}\right)+\psi\left(\frac{s-\rho_{0}}{2}+1\right)\right\}\right] .
\end{aligned}
$$

(iv) Let $\operatorname{Re}(s)>\rho_{0}$. Then we have

$$
\begin{aligned}
& \left(\Omega-s^{2}+\rho_{0}^{2}\right) \phi_{s}^{[r+1]}=-\phi_{s}^{[r]}, \quad r \in \mathbf{N}_{0} \\
& \left(\Omega-s^{2}+\rho_{0}\right) \phi_{s}^{[0]}=\delta
\end{aligned}
$$

in the sense of distributions on $G / K$ with $\delta$ the Dirac delta supported at the origin of $G / K$.

Proof.

(i) By the explicit formula of $\phi_{s}^{(2)}\left(\exp \left(t H_{0}\right)\right)=u_{s}^{(2)}(t)$, it follows that $\phi_{s}^{[r]}\left(\exp \left(t H_{0}\right)\right)=\left(-\frac{1}{2 s} \frac{d}{d s}\right)^{r} u_{s}^{(2)}(t)$ is $C^{\infty}$ on $t>0$ and $\phi_{s}^{[r]}\left(\exp \left(t H_{0}\right)\right)=$ $\phi_{s}^{[r]}\left(\exp \left(-t H_{0}\right)\right)$. From this, the assertion follows.

(ii) Since ${ }_{2} F_{1}\left(\frac{s+\rho_{0}}{2}, \frac{s-\rho_{0}}{2}+m ; s+1 ; z\right)$ is holomorphic with respect to $(s, z)$ on the domain $\{(s, z)|\operatorname{Re}(s)>0| z \mid,<1\}$, the function $z \mapsto\left(\frac{d}{d s}\right)^{j}{ }_{2} F_{1}\left(\frac{s+\rho_{0}}{2}, \frac{s-\rho_{0}}{2}+\right.$ 
$m ; s+1 ; z)$ is holomorphic on $|z|<1$, especially bounded at $z=0$. It is easy to see that $\phi_{s}^{[r]}\left(\exp \left(t H_{0}\right)\right)$ is expressed as a finite sum of the functions of the form

$$
d_{j}(s) \cdot(\cosh t)^{-\left(s+\rho_{0}\right)} \cdot\left(\frac{d}{d s}\right)_{2}^{j} F_{1}\left(\frac{s+\rho_{0}}{2}, \frac{s-\rho_{0}}{2}+m ; s+1 ; \frac{1}{\cosh ^{2} t}\right)
$$

with $0 \leqslant j \leqslant r$ and $d_{j}(s)$ a holomorphic function on $\operatorname{Re}(s)>0$. Therefore we have

$$
\phi_{s}^{[r]}\left(\exp \left(t H_{0}\right)\right)=O\left(e^{-\left(\operatorname{Re}(s)+\rho_{0}\right) t}\right) \quad(\text { as } t \rightarrow+\infty)
$$

(iii) By the formula of $Y_{s}(t)$, we have

$$
\begin{aligned}
\left(-\frac{1}{2 s} \frac{d}{d s}\right)^{r} \mathrm{Y}_{s}(t)= & \sum_{j=0}^{m-1} \frac{1}{(\sinh t)^{2 j}}\left(-\frac{1}{2 s} \frac{d}{d s}\right)^{r} \mathrm{a}_{j}(s) \\
& +\left(-\frac{1}{2 s} \frac{d}{d s}\right)^{r} \mathrm{~b}(s) \log \left(\sinh ^{2} t\right)+y(s)
\end{aligned}
$$

with some $y(s)$ independent of $t$. Since $\mathrm{a}_{j}(s)$ and $\mathrm{b}(s)$ are even polynomial functions of degree no more than $2(m-1),\left(-\frac{1}{2 s} \frac{d}{d s}\right)^{r} a_{j}(s)=0$ for $j=0, \ldots, m-1$ and $\left(-\frac{1}{2 s} \frac{d}{d s}\right)^{r} \mathrm{~b}(s)=0$ if $r \geqslant m$. Hence $\left(-\frac{1}{2 s} \frac{d}{d s}\right)^{r} Y_{s}(t)$ becomes smooth at $t=0$ as well as on $\mathbf{R}-\{0\}$. Since

$$
\phi_{s}^{[r]}\left(\exp \left(t H_{0}\right)\right)-\frac{c_{G}^{-1}}{(2-2 m) r !}\left(-\frac{1}{2 s} \frac{d}{d s}\right)^{r} \mathrm{Y}_{s}(t)
$$

is continuous in $t$ on all of $\mathbf{R}$ (Proposition 3.1.3 (b)), $\phi_{s}^{[r]}\left(\exp \left(t H_{0}\right)\right)$ has a continuous extention to all of $\mathbf{R}$ if $r \geqslant m$. This establishes the first part of (iii). The formula in (iii) follows from the explicit formula of $Y_{s}(t)$ in Proposition 3.1.3. that

(iv) If $\mu \geqslant 0$, then we set $C_{\mu}^{\infty}(G)$ equal to the all functions $f \in C^{\infty}(G)$ such

$$
p_{X, \mu}(f)=\sup _{g \in G}\|g\|^{-\mu}|X f(g)|<\infty
$$

for all $X \in U(\mathfrak{g})$. We endow $C_{\mu}^{\infty}(G)$ with the topology induced by the seminorms $p_{X, \mu}$. (see [17, page 675].) The argument below is similar to the proof of $[17$, Lemma 2.2]. Let $f \in C_{\mu}^{\infty}(G / K)$ with $\mu>0$, and $\operatorname{Re}(s)>\rho_{0}+\mu$. By the integration formula in Lemma 2.3.1, we have

$$
\begin{aligned}
& c_{G}^{-1} \int_{G} \phi_{s}^{[r]}\left(x^{-1} y\right) \cdot\left(\left(\Omega-s^{2}+\rho_{0}^{2}\right) f\right)(y) d y \\
= & \int_{K} \int_{0}^{+\infty} \int_{K} \phi_{s}^{[r]}\left(k_{1} \exp \left(t H_{0}\right) k_{2}\right) \cdot\left(\left(\Omega-s^{2}+\rho_{0}^{2}\right) f\right)\left(x \cdot k_{1} \exp \left(t H_{0}\right) k_{2}\right) \mu(t) d k_{1} d t d k_{2} \\
= & \int_{0}^{+\infty} \phi_{s}^{[r]}\left(\exp \left(t H_{0}\right)\right) \cdot\left(\left(\Omega-s^{2}+\rho_{0}^{2}\right) F\right)\left(\exp \left(t H_{0}\right)\right) \mu(t) d t
\end{aligned}
$$


with $F(g)=\int_{K} f(x k g) d k \quad(g \in G)$. For $t>0$, put $h_{r}(t)=\phi_{s}^{[r]}\left(\exp \left(t H_{0}\right)\right)$ and $F_{0}(t)=F\left(\exp \left(t H_{0}\right)\right)$. Then by integration by parts, the last formula of (3.26) becomes

$$
\begin{aligned}
& \int_{0}^{+\infty} h_{r}(t) \cdot D_{t} F_{0}(t) \cdot \mu(t) d t \\
= & \lim _{\zeta \rightarrow \infty} \lim _{\epsilon \rightarrow+0}\left\{\mu(\zeta) h_{r}(\zeta) F_{0}^{\prime}(\zeta)-\mu(\zeta) h_{r}^{\prime}(\zeta) F_{0}(\zeta)-\mu(\epsilon) h_{r}(\epsilon) F_{0}^{\prime}(\epsilon)+\mu(\epsilon) h_{r}^{\prime}(\epsilon) F_{0}(\epsilon)\right\} \\
& \quad+\int_{0}^{+\infty} D_{t} h_{r}(t) \cdot F_{0}(t) \cdot \mu(t) d t
\end{aligned}
$$

with $D_{t}=\mu(t)^{-1} \frac{d}{d t}\left(\mu(t) \frac{d}{d t}\right)+\rho_{0}^{2}-s^{2}$. By Proposition 3.1.5 (i), we have $h_{r}(t) \prec$ $e^{-\left(\operatorname{Re}(s)+\rho_{0}\right) \zeta}$ as $\zeta \rightarrow+\infty$ and $h_{r}^{\prime}(t) \prec e^{-\left(\operatorname{Re}(s)+\rho_{0}\right) \zeta}$ as $\zeta \rightarrow+\infty$. By the formula of $\mu(t)$, we have, $\mu(\zeta) \prec e^{2 \rho_{0} s}$ as $\zeta \rightarrow+\infty$. Using the property of the norm $\|\cdot\|$, we have some constants $T$ and $M_{0}>0$ such that

$$
\left\|x k \exp \left(t H_{0}\right)\right\| \leqslant\|x\| \cdot\left\|\exp \left(t H_{0}\right)\right\| \leqslant M_{0}\|x\| e^{t} \quad(\forall t \geqslant T, \forall k \in K) .
$$

Since $f \in C_{\mu}^{\infty}(G / K)$, we thus have

$$
\begin{aligned}
& \left|F_{0}(\zeta)\right| \leqslant p_{\mu}(f) \cdot M_{0}\|x\| e^{\zeta \mu} \quad(\forall \zeta \geqslant T), \\
& \left|F_{0}^{\prime}(\zeta)\right| \leqslant p_{H_{0}, \mu}(f) \cdot M_{0}\|x\| e^{\zeta \mu} \quad(\forall \zeta \geqslant T) .
\end{aligned}
$$

Therefore the first and the second terms in the bracket of the right-hand side of (3.27) is estimated as

$$
\begin{aligned}
& \left|\mu(\zeta) h_{r}(\zeta) F_{0}{ }^{\prime}(\zeta)\right| \prec e^{\left(-\operatorname{Re}(s)+\rho_{0}+\mu\right) \zeta} \\
& \left|\mu(\zeta) h_{r}{ }^{\prime}(\zeta) F_{0}(\zeta)\right| \prec e^{\left(-\operatorname{Re}(s)+\rho_{0}+\mu\right) \zeta}
\end{aligned}
$$

so they tend to zero as $\zeta \rightarrow+\infty$. We evaluate the third and the fourth term in the right-hand side of (3.27). By the expression of $\phi_{s}^{[r]}\left(\exp \left(t H_{0}\right)\right)$ around $t=0$ given in Proposition 3.1.3, we have

$$
\begin{aligned}
& \left|h_{r}(\epsilon)\right| \prec \epsilon^{-2(m-1)} \quad(\epsilon \rightarrow+0), \\
& h_{r}^{\prime}(\epsilon)=\frac{c_{G}^{-1}}{\epsilon^{2 m-1}}(1+O(\epsilon \log \epsilon)) \quad(\text { if } r=0), \\
& \left|h_{r}^{\prime}(\epsilon)\right| \prec \epsilon^{-(2 m-2 r-1)} \quad(\text { if } r>0) .
\end{aligned}
$$

Since $\mu(\epsilon) \prec \epsilon^{p+q}=\epsilon^{2 m-1}$ and

$$
F_{0}(\epsilon)=F_{0}(0)+O(\epsilon), \quad F_{0}^{\prime}(\epsilon) \prec 1
$$

We have

$$
\begin{aligned}
& \left|\mu(\epsilon) h_{r}(\epsilon) F_{0}^{\prime}(\epsilon)\right| \prec \epsilon \quad(\epsilon \rightarrow+0), \\
& \left|\mu(\epsilon) h_{r}^{\prime}(\epsilon) F_{0}(\epsilon)\right| \prec \epsilon^{2 r} \quad(\epsilon \rightarrow+0) \text { if } r>0, \\
& \mu(\epsilon) h_{r}^{\prime}(\epsilon) F_{0}(\epsilon)=c_{G}^{-1}(1+O(\epsilon \log \epsilon)) F_{0}(0) \quad(r=0) .
\end{aligned}
$$

Therefore, the third and the fourth term of (3.27) are equal to zero if $r=1$ and are equal to $c_{G}^{-1} F_{0}(0)$ if $r=0$. Suppose $r=0$. Since the last integral in the right-hand 
side of (3.27) vanishes due to the differential equation $D_{t} h(t)=0(t>0)$, we have

$$
\begin{aligned}
& c_{G}^{-1} \int_{G} \phi_{s}^{[0]}\left(x^{-1} y\right)\left(\left(\Omega-s^{2}+\rho_{0}^{2}\right) f\right)(y) d y \\
= & F_{0}(0) c_{G}^{-1}=f(x) c_{G}^{-1}
\end{aligned}
$$

When $r>1$, all the terms in the right-hand side of (3.27) except the last one vanish. Consequently we have

$$
\begin{aligned}
& c_{G}^{-1} \int_{G} \phi_{s}^{[r]}\left(x^{-1} y\right)\left(\left(\Omega-s^{2}+\rho_{0}^{2}\right) f\right)(y) d y \\
= & \int_{0}^{\infty} D_{t} h_{r}(t) \cdot F_{0}(t) \cdot \mu(t) d t
\end{aligned}
$$

To compute $D_{t} h_{r}(t)$, we need a formula:

CLAIM 3.1.6. For $r \geqslant 1$, we have

$$
\left[D_{t},\left(-\frac{1}{2 s} \frac{d}{d s}\right)^{r}\right]=-r\left(-\frac{1}{2 s} \frac{d}{d s}\right)^{r-1}
$$

Proof of the claim. Obviously it is sufficient to prove

$$
\left[s^{2},\left(-\frac{1}{2 s} \frac{d}{d s}\right)^{r}\right]=r\left(-\frac{1}{2 s} \frac{d}{d s}\right)^{r-1} .
$$

If $r=1$, then this is checked by a direct computation. Let $r>1$, and assume that the formula for $r-1$ is valid. Then,

$$
\begin{aligned}
& s^{2}\left(-\frac{1}{2 s} \frac{d}{d s}\right)^{r} \\
= & s^{2}\left(-\frac{1}{2 s} \frac{d}{d s}\right)^{r-1} \cdot\left(-\frac{1}{2 s} \frac{d}{d s}\right) \\
= & \left\{\left(-\frac{1}{2 s} \frac{d}{d s}\right)^{r-1} \cdot s^{2}+(r-1)\left(-\frac{1}{2 s} \frac{d}{d s}\right)^{r-2}\right\}\left(-\frac{1}{2 s} \frac{d}{d s}\right) \\
= & \left(-\frac{1}{2 s} \frac{d}{d s}\right)^{r-1} \cdot \frac{-s}{2} \frac{d}{d s}+(r-1)\left(-\frac{1}{2 s} \frac{d}{d s}\right)^{r-1} .
\end{aligned}
$$

To have the second equality, we used the induction-assumption. Since $-\frac{s}{2} \frac{d}{d s}=1+$ $\left(-\frac{1}{2 s} \frac{d}{d s}\right) s^{2}$, we obtain

$$
\begin{aligned}
& s^{2}\left(-\frac{1}{2 s} \frac{d}{d s}\right)^{r} \\
= & \left(-\frac{1}{2 s} \frac{d}{d s}\right)^{r-1}\left(1-\frac{1}{2 s} \frac{d}{d s} \cdot s^{2}\right)+(r-1)\left(-\frac{1}{2 s} \frac{d}{d s}\right)^{r-1} \\
= & r\left(-\frac{1}{2 s} \frac{d}{d s}\right)^{r-1}+\left(-\frac{1}{2 s} \frac{d}{d s}\right)^{r} \cdot s^{2} .
\end{aligned}
$$

This completes the proof of the formula for $r$. 
Let us finish the proof of the proposition. We apply the claim to the function $\phi_{s}^{(2)}\left(\exp \left(t H_{0}\right)\right)$ in $s$. Then

$$
\begin{aligned}
& D_{t} h_{r}(t) \\
= & D_{t}\left(-\frac{1}{2 s} \frac{d}{d s}\right)^{r} \frac{c_{G}^{-1}}{(2-2 m) r !} \phi_{s}^{(2)}\left(\exp \left(t H_{0}\right)\right) \\
= & \left\{-r\left(-\frac{1}{2 s} \frac{d}{d s}\right)^{r-1}+\left(-\frac{1}{2 s} \frac{d}{d s}\right)^{r} D_{t}\right\} \frac{c_{G}^{-1}}{(2-2 m) r !} \phi_{s}^{(2)}\left(\exp \left(t H_{0}\right)\right) \\
= & -h_{r-1}(t)+\frac{c_{G}^{-1}}{(2-2 m) r !}\left(-\frac{1}{2 s} \frac{d}{d s}\right)^{r} D_{t} \phi_{s}^{(2)}\left(\exp \left(t H_{0}\right)\right) .
\end{aligned}
$$

Since $D_{t} \phi_{s}^{(2)}\left(\exp \left(t H_{0}\right)\right)=0$ for $t>0$, we have

$$
D_{t} h_{r}(t)=-h_{r-1}(t) \quad \text { for } t>0 .
$$

By (3.32), (3.34) and (3.26), we consequently have

$$
\begin{aligned}
& c_{G}^{-1} \int_{G} \phi_{s}^{[r]}\left(x^{-1} y\right)\left(\left(\Omega-s^{2}+\rho_{0}^{2}\right) f\right)(y) d y \\
= & -c_{G}^{-1} \int_{G} \phi_{s}^{[r-1]}\left(x^{-1} y\right) \cdot f(y) d y
\end{aligned}
$$

for $r \geqslant 1$. By (3.31) and (3.35), we have the desired formula.

We have a characterization of the family $\left\{\phi_{s}^{[r]}\right\}$.

Proposition 3.1.7. Let $\left\{\varphi_{r, s} \mid r \in \mathbf{N}_{0}, \operatorname{Re}(s)>\rho_{0}\right\}$ be a family of bi-K-invariant distributions on $G$ with the following properties.

(i) For $r \in \mathrm{N}_{0}, \operatorname{Re}(s)>\rho_{0}$ the distribution $\varphi_{r, s}$ is represented by a $C^{\infty}$-function on $G^{+}$.

(ii) For $\operatorname{Re}(s)>\rho_{0}$,

$$
\lim _{t \rightarrow+0} t^{2 m-2} \varphi_{0, s}\left(\exp \left(t H_{0}\right)\right)=1
$$

(iii) For $r \in \mathbf{N}_{0}, \operatorname{Re}(s)>\rho_{0}$,

$$
\varphi_{r, s}\left(\exp \left(t H_{0}\right)\right)=O\left(e^{-t\left(\operatorname{Re}(s)+\rho_{0}\right)}\right), \quad t \rightarrow+\infty .
$$

(iv) Let $\operatorname{Re}(s)>\rho_{0}$. If we regard $\varphi_{r, s}$ 's as distributions on $G / K$, they satisfy the differential equations

$$
\begin{aligned}
& \left(\Omega-s^{2}+\rho_{0}^{2}\right) \varphi_{r+1, s}=-\varphi_{r, s}, \quad r \in \mathbf{N}_{0} \\
& \left(\Omega-s^{2}+\rho_{0}^{2}\right) \varphi_{0, s}=\delta
\end{aligned}
$$

Then for $r \in \mathbf{N}_{0}$ and $s \in \mathbf{C}, \operatorname{Re}(s)>\rho_{0}$ we have $\varphi_{r, s}(g)=\phi_{s}^{[r]}(g)$ on $G / K$ in the sense of distributions.

Proof. The difference $\varphi_{0, s}-\phi_{s}^{[0]}$, considered to be a distribution on $G / K$, is annihilated by the elliptic differential operator $\Omega-s^{2}+\rho_{0}^{2}$ on $G / K$ (see Proposition 3.1.5 (iv)). Hence by the elliptic regularity theorem $\varphi_{0, s}-\phi_{s}^{[0]}$ is smooth on all of $G$. By Proposition 3.1.1 (ii) the two distributions $\varphi_{0, s}$ and $\phi_{s}^{[0]}$ coincide with each other on 
the open dense set $G^{+}$. Therefore $\varphi_{0, s}=\phi_{s}^{[0]}$ on all of $G$. Let $r>0$. We use induction on $r$ to show $\varphi_{r, s}=\phi_{s}^{[r]}$. Assume $\varphi_{r-1, s}=\phi_{s}^{[r-1]}$. Then from the property (iv) of $\varphi_{r, s}$ and Proposition 3.1.4 (iv), the distribution $\varphi_{r, s}-\phi_{s}^{[r]}$ (on $G / K$ ) is annihilated by the elliptic differential operator $\Omega-s^{2}+\rho_{0}^{2}$; hence it becomes smooth on all of $G$ and is actually a constant (say $C_{0}$ ) multiple of the zonal spherical function $\phi_{s}$. But the property (iii) and Proposition 3.1.5 (ii) give that $\left(\varphi_{r, s}-\phi_{s}^{[r]}\right)\left(\exp \left(t H_{0}\right)\right)$ is of exponential decay as $t \rightarrow+\infty$. Since $\phi_{s}\left(\exp \left(t H_{0}\right)\right)$ with $\operatorname{Re}(s)>\rho_{0}$ grows exponentially as $t \rightarrow+\infty$, the proportionality constant $C_{0}$ should be zero. This completes the proof.

3.2. Miatello-Wallach's spherical functions. We recall some basic properties of the functions $Q_{r, s}, r \in \mathbf{N}$ which Miatello-Wallach introduced and studied in detail ([17]).

(i) For $s \in \mathbf{C}, \operatorname{Re}(s)>0, Q_{1, s} \in C^{\infty}\left(K \backslash G^{+} / K\right)$ ([17, Theorem 1.1 (a)]) .

(ii) For a fixed $g \in G^{+}$, the function $s \mapsto Q_{1, s}(g)$ is holomorphic on $\operatorname{Re}(s)>0$ and has a meromorphic continuation to $\mathbf{C}([17$, Theorem $1.1,(\mathrm{~b})])$.

(iii)

$$
Q_{1, s}\left(\exp \left(t H_{0}\right)\right) \sim \frac{c_{G}^{-1} s c(s)}{m-1} \cdot t^{2-2 m}, \quad t \rightarrow+0
$$

([17, Theorem 1.1, (d)]).

(iv) Let $\operatorname{Re}(s)>\rho_{0}$ and $r \in \mathbf{N}$. Then $Q_{r, s}$ is bi- $K$-invariant and integrable function on $G$ satisfying the formula

$$
Q_{r+1, s}=Q_{1, s} \star Q_{r, s}
$$

Here $\star$ means the convolution on $G$ with respect to the measure $d g$. (see $[17$, page 678]).

(v) Let $\operatorname{Re}(s)>\rho_{0}$ and $r \in \mathbf{N}$. Then

$$
Q_{r, s}\left(\exp \left(t H_{0}\right)\right)=O\left(e^{-t\left(\operatorname{Re}(s)+\rho_{0}\right)}\right), \quad t \rightarrow+\infty
$$

([17, Lemma 2.4]).

(vi) Let $\operatorname{Re}(s)>\rho_{0}$. Then the distributions $Q_{r, s}$ on $G / K$ satisfy the differential equations

$$
\left(\Omega-s^{2}+\rho_{0}^{2}\right) Q_{r+1, s}=-2 s c(s) Q_{r, s}
$$

for $r \in \mathbf{N}_{0}$ with the convention that $Q_{0, s}=\delta$, the Dirac delta supported at the origin of $G / K([17$, Lemma 2.2, Lemma 2.6]).

Thus the family $\left\{-(2 s c(s))^{-(r+1)} Q_{r+1, s} \mid r \in \mathbf{N}_{0}, \operatorname{Re}(s)>\rho_{0}\right\}$ possesses all the properties (i) to (iv) in Proposition 3.1.7. Hence applying that proposition, we have the following theorem, which is one of the main results of this article.

Theorem 3.2.1. Let $\operatorname{Re}(s)>\rho_{0}$ and $r \in \mathrm{N}_{0}$. Then as distributions on $G / K$ the equality

$$
\phi_{s}^{[r]}(g)=-\left(\frac{1}{2 s c(s)}\right)^{r+1} Q_{r+1, s}(g)
$$

holds. 


\section{Miatello-Wallach's function $\mathbf{P}_{r, s}$ and its spectral expansion.}

4.1. The function $\mathbf{P}_{r, s}$. Let $X=G / K$. Let $\Gamma$ be a neat co-finite lattice of $G$, that is a discrete torsion-free subgroup of $G$ such that $\Gamma \backslash G$ has finite volume. We assume that if $\Gamma$ is not cocompact then it satisfies the Langlands' axiom. Here is a notational convention: A point of the double coset space $\Gamma \backslash X$ is denoted by a letter with a dot and any one of the lifts of that point to $G$ is by the same letter without a dot. For example if $x \in G$ then the corresponding coset $\Gamma x K \in \Gamma \backslash X$ is $\dot{x}$.

Let $\triangle$ be the Laplacian of $\Gamma \backslash X$ corresponding to $-\Omega$.

In [17], Miatello-Wallach introduced the functions $\mathbf{P}_{r, s}\left(r \in \mathbf{N}_{0}, \operatorname{Re}(s)>\rho_{0}\right)$ by

$$
\mathbf{P}_{r, s}(\dot{x}, \dot{y})=-\left(\frac{1}{2 s c(s)}\right)^{r} \sum_{\gamma \in \Gamma} Q_{r, s}\left(x^{-1} \gamma y\right), \quad \dot{x}, \dot{y} \in \Gamma \backslash X
$$

with $Q_{r, s}$ the spherical function which we recalled in 3.2. Among other things, they proved that

(a) the series $\mathbf{P}_{r, s}(\dot{x}, \dot{y})$ converges absolutely and defines $\mathbf{P}_{r, s}(\dot{x}, \dot{y})$ holomorphic in $s$ on $\operatorname{Re}(s)>\rho_{0}$ and smooth in $\dot{x}, \dot{y}$ in the complement of the diagonal of $(\Gamma \backslash X) \times(\Gamma \backslash X)$

(b) for each $\dot{x} \in \Gamma \backslash X$, as a distribution on $\Gamma \backslash X \mathbf{P}_{r, s}(\dot{x},-)$ satisfies

$$
\left(\triangle+s^{2}-\rho_{0}^{2}\right)^{r} \mathbf{P}_{r, s}(\dot{x},-)=-\delta(\dot{x})
$$

with $\delta(\dot{x})$ the Dirac delta on $\Gamma \backslash X$ supported at $\dot{x}$ ([17, page 685, Theorem 3.4], [2, page 621, Theorem 3.2]).

Proposition 4.1.1. Let $s \in \mathbf{C}$ with $\operatorname{Re}(s)>\rho_{0}$. If $r>m$, then $\mathbf{P}_{r, s}(\dot{x}, \dot{y})$ has a unique continuous extension to all of $(\Gamma \backslash X) \times(\Gamma \backslash X)$.

Proof. This is a consequence of [17, Corollary 2.5]. Indeed, by that corollary, $Q_{r, s} \in L^{2}(G)$ if $r>m / 2$ and $\operatorname{Re}(s)>\rho_{0}$. Hence $Q_{r, s}$ is continuous on all of $G$ if $r>m$ and $\operatorname{Re}(s)>\rho_{0}$, since in general the convolution of two $L^{2}$-functions on $G$ gives a continuous function. By the discussion in [17, pp.683-687], the continuity of $\mathbf{P}_{r, s}$ follows.

REMARK. Proposition 4.1.1 is also a corollary of our earlier results. Indeed, from Proposition 3.1.5 (iii) and Theorem 3.2.1, the function $Q_{r, s}\left(r>m, \operatorname{Re}(s)>\rho_{0}\right)$ has a continuous extension on $G$.

By this proposition, we can consider the restriction of $\mathbf{P}_{r, s}(\dot{x}, \dot{y})$ to the diagonal $\dot{x}=\dot{y}$ of $(\Gamma \backslash X) \times(\Gamma \backslash X)$. From now on we assume that $\Gamma$ is cocompact. Then $\mathbf{P}_{r, s}(\dot{x}, \dot{y})$ becomes bounded on $(\Gamma \backslash X) \times(\Gamma \backslash X)$ if $r>m$; in particular the function $\mathbf{P}_{r, s}(\dot{x}, \dot{x})$ is integrable on $\Gamma \backslash X$. We want to evaluate the integral

$$
\int_{\Gamma \backslash X} \mathbf{P}_{r+1, s}(\dot{x}, \dot{x}) d \dot{x}
$$

with $r \geqslant m$ explicitly.

4.2. Spectral expansion of $\mathbf{P}_{r, s}(\dot{x}, \dot{y})$. In this subsection we compute the integral (4.1) by using the spectral expansion of $\mathbf{P}_{r+1, s}(\dot{x}, \dot{y})$. Since we assume that $\Gamma$ is cocompact the Laplacian $\triangle$ has no continuous spectrum on $L^{2}(\Gamma \backslash X)$. The eigenvalues of $\triangle$ forms a countable subset of non-negative reals enumerated as

$$
0=\lambda_{0}<\lambda_{1} \leqslant \lambda_{2} \leqslant \ldots \leqslant \lambda_{n} \leqslant \ldots
$$


so that each eigenvalue occurs in this sequence with its multiplicity. Let $\left\{\varphi_{n}\right\}_{n \geqslant 0}$ be the orthonormal basis of $L^{2}(\Gamma \backslash X)$ such that $\varphi_{n} \in C^{\infty}(\Gamma \backslash X)$ and $\Delta \varphi_{n}=\lambda_{n} \varphi_{n}$. For each $n$ we fix a complex number $s_{n}$ such that $\lambda_{n}=\rho_{0}^{2}-s_{n}^{2}$.

Proposition 4.2.1. Let $r \in \mathbf{N}_{0}$ and $s \in \mathbf{C}$ be such that $r \geqslant m$ and $\operatorname{Re}(s)>\rho_{0}$. Then

$$
\mathbf{P}_{r+1, s}(\dot{x}, \dot{y})=-\sum_{n=0}^{\infty} \frac{\overline{\varphi_{n}(\dot{x})} \varphi_{n}(\dot{y})}{\left(s^{2}-s_{n}^{2}\right)^{r+1}}, \quad \dot{x}, \dot{y} \in \Gamma \backslash X .
$$

Here the infinite series in the right-hand side of this identity converges uniformly in $(\dot{x}, \dot{y}) \in(\Gamma \backslash X) \times(\Gamma \backslash X)$.

Proof. Since $r+1>m$ and $\Gamma \backslash X$ is compact, [17, Theorem 4.2, page 689] implies that the series

$$
\sum_{n=0}^{\infty} \frac{\left|\varphi_{n}(\dot{x})\right|^{2}}{\left|s^{2}-s_{n}^{2}\right|^{r+1}}
$$

converges uniformly in $\dot{x} \in \Gamma \backslash X$. Hence by Cauchy-Schwarz inequality, we have that the series

$$
\Phi(\dot{x}, \dot{y})=-\sum_{n=0}^{\infty} \frac{\overline{\varphi_{n}(\dot{x})} \varphi_{n}(\dot{y})}{\left(s^{2}-s_{n}^{2}\right)^{r+1}}
$$

converges absolutely and uniformly in $(\dot{x}, \dot{y}) \in(\Gamma \backslash X)^{2}$, which in turn means the continuity of the function $\Phi(\dot{x}, \dot{y})$ on $(\Gamma \backslash X)^{2}$. Since $\Gamma \backslash X$ is compact, for a fixed $\dot{x}$, the function $\Phi(\dot{x},-)$ is bounded on $\Gamma \backslash X$; in particular it is in $L^{2}(\Gamma \backslash X)$. Moreover

$$
\left\langle\Phi(\dot{x},-), \varphi_{n}\right\rangle=-\frac{\overline{\varphi_{n}(\dot{x})}}{\left(s^{2}-s_{n}^{2}\right)^{r+1}}
$$

for $n \in \mathbf{N}_{0}$. Since the last formula equals $\left\langle\mathbf{P}_{r, s}(\dot{x},-), \varphi_{n}\right\rangle$ ([17, page 688]), the difference $\Phi(\dot{x},-)-\mathbf{P}_{r, s}(\dot{x},-)$ is orthogonal to all $\varphi_{n}$. Noting that the difference is continuous on $\Gamma \backslash X$ (Proposition 4.1.1) we indeed have $\Phi(\dot{x}, \dot{y})=\mathbf{P}_{r, s}(\dot{x}, \dot{y})$ for all $\dot{y}$. of $\triangle$.

By this proposition we can compute the integral (4.1) in terms of the eigenvalues

Proposition 4.2.2. If $r \geqslant m$ and $\operatorname{Re}(s)>\rho_{0}$, then

$$
\int_{\Gamma \backslash X} \mathbf{P}_{r+1, s}(\dot{x}, \dot{x}) d \dot{x}=-\sum_{n=0}^{\infty} \frac{1}{\left(s^{2}-s_{n}^{2}\right)^{r+1}} .
$$

Proof. This is a direct consequence of Proposition 4.2.1

5. Computation of the integral $\int_{\Gamma \backslash X} \mathbf{P}_{r, s}(\dot{x}, \dot{x}) d \dot{x}$ and the resolvent trace formula. 
5.1. Computation of hyperbolic term. Let $\Gamma$ be as in the previous section. Then an element $\gamma \in \Gamma-\{e\}$ is $G$-conjugate to an element $h_{\gamma}$ of $A^{+} M$ with $A^{+}=$ $\exp \left((0,+\infty) H_{0}\right)$ and $M$ the centralizer of $A$ in $K ; h_{\gamma}$ is not uniquely determined by $\gamma$, but its ambiguity is unimportant for our purpose. We can write

$$
h_{\gamma}=\exp \left(t_{\gamma} H_{0}\right) m_{\gamma}, \quad t_{\gamma}>0, m_{\gamma} \in M .
$$

Let $G_{\gamma}$ be the centralizer of $\gamma$ in $G$ and put $\Gamma_{\gamma}=\Gamma \cap G_{\gamma}$. Then $G_{\gamma}$ is reductive and $\Gamma_{\gamma} \backslash G_{\gamma}$ is compact. We fix a Haar measure $d g_{\gamma}$ on $G_{\gamma}$ in a manner analogous to the manner in which the Haar measure on $G$ was fixed, following the Iwasawa decomposition of $G_{\gamma}$, and put $d \dot{g}_{\gamma}$ for the invariant measure on $\Gamma_{\gamma} \backslash G_{\gamma}$. The group $\Gamma_{\gamma}$ is known to be isomorphic to $\mathbf{Z}$. Hence there exists a unique generator $\gamma_{0}$ of $\Gamma_{\gamma}$ and a positive integer $j(\gamma)$ (the multiplicity of $\gamma$ ) such that $\gamma=\gamma_{0}^{j(\gamma)}$. Let $\mathcal{H}(\Gamma)$ be the set of $\Gamma$-conjugacy classes in $\Gamma-\{e\}$. We first calculate the orbital integral of $\phi_{s}^{[r]}$ associated with a hyperbolic conjugacy class.

Proposition 5.1.1. Let $r \in \mathbf{N}_{0}$ and $\operatorname{Re}(s)>\rho_{0}$. For $[\gamma] \in \mathcal{H}(\Gamma)$, put

$$
J^{[r]}([\gamma] ; s)=\operatorname{vol}\left(\Gamma_{\gamma} \backslash G_{\gamma}\right) \int_{G_{\gamma} \backslash G} \phi_{s}^{[r]}\left(g^{-1} \gamma g\right) d g_{\gamma}^{*}
$$

where $d g_{\gamma}^{*}$ is the $G$-invariant measure on $G_{\gamma} \backslash G$ normalized so that $d g=d g_{\gamma} d g_{\gamma}^{*}$. Then the integral $J^{[r]}([\gamma] ; s)$ converges absolutely and uniformly on $\operatorname{Re}(s) \geqslant \rho_{0}+\epsilon$ for any $\epsilon>0$ and is evaluated as

$$
J^{[r]}([\gamma] ; s)=\frac{1}{r !}\left(-\frac{1}{2 s} \frac{d}{d s}\right)^{r}\left\{-j(\gamma)^{-1} \mid \operatorname{det}\left(1-\left.\left.\operatorname{Ad}\left(h_{\gamma}\right)^{-1}\right|_{\mathfrak{n})}\right|^{-1} \frac{t_{\gamma} e^{-\left(s+\rho_{0}\right) t_{\gamma}}}{2 s}\right\} .\right.
$$

Proof. We shall compute the orbital integral

$$
I^{[r]}([\gamma] ; s)=\int_{G_{\gamma} \backslash G} \phi_{s}^{[r]}\left(g^{-1} \gamma g\right) d g_{\gamma}^{*}
$$

Let $h=h_{\gamma}=\exp \left(t_{\gamma} H_{0}\right) m_{\gamma}=\exp \left(t H_{0}\right) m \in A^{+} M$, to which $\gamma$ is conjugate. Then $I^{[r]}([\gamma] ; s)=I^{[r]}([h] ; s)$ and

$$
I^{[r]}([h] ; s)=\int_{G_{h} \backslash G} \phi_{s}^{[r]}\left(g^{-1} h g\right) d g_{h}^{*}=\operatorname{vol}\left(A \backslash G_{h}\right)^{-1} \int_{A \backslash G} \phi_{s}^{[r]}\left(g^{-1} h g\right) \frac{d g}{d a},
$$

where $\operatorname{vol}\left(A \backslash G_{h}\right)=\int_{A \backslash G_{h}} \frac{d g_{h}}{d a}$. Moreover

$$
\begin{aligned}
& \int_{A \backslash G} \phi_{s}^{[r]}\left(g^{-1} h g\right) \frac{d g}{d a}=\int_{N} \int_{K} \phi_{s}^{[r]}\left(k^{-1} n^{-1} h n k\right) d k d n \\
= & \int_{N} \phi_{s}^{[r]}\left(n^{-1} h n\right) d n=\left|\operatorname{det}\left(1-\left.\operatorname{Ad}(h)\right|_{\mathfrak{n}}\right)\right|^{-1} \int_{N} \phi_{s}^{[r]}(n h) d n .
\end{aligned}
$$

We recall a lemma (cf. $\left[12\right.$, p.487]) to calculate the integral (5.3). Put $|Z|^{2}=$ $-B(X, \theta X)$ for $Z \in \mathfrak{g}$. If $n=\exp (X+Y)$ with $X \in \mathfrak{n}_{\beta}, Y \in \mathfrak{n}_{2 \beta}$ and we write

$$
n h=n \cdot \exp \left(t H_{0}\right) m=k_{1} \exp \left(u H_{0}\right) k_{2} \quad\left(k_{1}, k_{2} \in K, u>0\right),
$$

then we have

$$
\cosh ^{2}(u)=\left[\cosh (t)+\frac{c}{2} e^{-t}|X|^{2}\right]^{2}+c e^{-2 t}|Y|^{2}
$$


where $c=4^{-1}(p+4 q)^{-1}=2^{-1} c_{0}$. We first treat the case of $r=0$. We have

$$
\begin{aligned}
& \int_{N} \phi_{s}^{[0]}(n h) d n \\
= & \int_{N} \phi_{s}^{[0]}\left(k_{1} \exp \left(u H_{0}\right) k_{2}\right) d n \\
= & \frac{c_{G}^{-1}}{(2-2 m)} \int_{\mathfrak{n}_{\beta} \times \mathfrak{n}_{2 \beta}} \phi_{s}^{(2)}\left(\exp u H_{0}\right) d X d Y \\
= & \frac{c_{G}^{-1}}{(2-2 m)} \gamma(s) \int_{\mathfrak{n}_{\beta} \times \mathfrak{n}_{2 \beta}}(\cosh u)^{-\left(s+\rho_{0}\right)}{ }_{2} F_{1}\left(\alpha, \beta ; s+1 ; \frac{1}{\cosh ^{2} u}\right) d X d Y \\
= & \frac{c_{G}^{-1}}{(2-2 m)} \gamma(s) \int_{\mathfrak{n}_{\beta} \times \mathfrak{n}_{2 \beta}}\left\{\left(\cosh (t)+\frac{c}{2} e^{-t}|X|^{2}\right)^{2}+c e^{-2 t}|Y|^{2}\right\}^{-\frac{s+\rho_{0}}{2}} \\
& \times_{2} F_{1}\left(\alpha, \beta ; s+1 ; \frac{1}{\left(\cosh (t)+\frac{c}{2} e^{-t}|X|^{2}\right)^{2}+c e^{-2 t}|Y|^{2}}\right) d X d Y,
\end{aligned}
$$

where $\alpha=\left(s+\rho_{0}\right) / 2$ and $\beta=\left(s-\rho_{0}+2 m\right) / 2$. Substituting $X=\cosh (t)^{\frac{1}{2}}\left(2^{-1} c\right)^{-\frac{1}{2}} e^{\frac{t}{2}} \xi$ and $Y=\cosh (t) c^{-\frac{1}{2}} e^{t} \eta$, we have $d X=\left(\cosh (t)^{\frac{1}{2}}\left(2^{-1} c\right)^{-\frac{1}{2}} e^{\frac{t}{2}}\right)^{p} d \xi, d Y=$ $\left(\cosh (t) c^{-\frac{1}{2}} e^{t}\right)^{q} d \eta$ and $d X d Y=(\cosh t)^{\rho_{0}} e^{\rho_{0} t} 2^{-\frac{p}{2}} c^{-m+\frac{1}{2}} d \xi d \eta$. So the integral (5.6) equals

$$
\begin{aligned}
& \frac{c_{G}^{-1}}{(2-2 m)} \gamma(s) \int_{\mathbf{R}^{p} \times \mathbf{R}^{q}}\left\{\left(1+\|\xi\|^{2}\right)^{2}+\|\eta\|^{2}\right\}^{-\frac{s+\rho_{0}}{2}} \cdot(\cosh t)^{-\left(s+\rho_{0}\right)+\rho_{0}} \\
& \times{ }_{2} F_{1}\left(\alpha, \beta ; s+1 ; \frac{(\cosh t)^{-2}}{\left(1+\|\xi\|^{2}\right)^{2}+\|\eta\|^{2}}\right) e^{\rho_{0} t} 2^{\frac{p}{2}} c^{-m+\frac{1}{2}} d \xi d \eta \\
= & \frac{c_{G}^{-1}}{(2-2 m)} \gamma(s)(\cosh t)^{-s} e^{\rho_{0} t} 2^{\frac{p}{2}} c^{-m+\frac{1}{2}} I(s) .
\end{aligned}
$$

Here, $I(s)$ is given by

$$
\int_{\mathbf{R}^{p} \times \mathbf{R}^{q}}\left\{\left(1+\|\xi\|^{2}\right)^{2}+\|\eta\|^{2}\right\}_{2}^{-\frac{s+\rho_{0}}{2}} F_{1}\left(\alpha, \beta ; s+1 ; \frac{(\cosh t)^{-2}}{\left(1+\|\xi\|^{2}\right)^{2}+\|\eta\|^{2}}\right) d \xi d \eta
$$

Using polar coordinates for $\mathbf{R}^{p}, \mathbf{R}^{q}$ and the power series expansion of the hypergeometric function, we carry out the integral term by term; this is permissible by dominated convergence theorem. Hence,

$$
\begin{aligned}
I(s)= & \frac{2 \pi^{\frac{p}{2}}}{\Gamma\left(\frac{p}{2}\right)} \frac{2 \pi^{\frac{q}{2}}}{\Gamma\left(\frac{q}{2}\right)} \int_{0}^{\infty} \int_{0}^{\infty} \rho_{1}^{p-1} \rho_{2}^{q-1}\left\{\left(1+\rho_{1}^{2}\right)^{2}+\rho_{2}^{2}\right\}^{-\frac{s+\rho_{0}}{2}} \\
& \times{ }_{2} F_{1}\left(\alpha, \beta ; s+1 ; \frac{(\cosh t)^{-2}}{\left(1+\rho_{1}^{2}\right)^{2}+\rho_{2}^{2}}\right) d \rho_{1} d \rho_{2} \\
= & \frac{2 \pi^{\frac{p}{2}}}{\Gamma\left(\frac{p}{2}\right)} \frac{2 \pi^{\frac{q}{2}}}{\Gamma\left(\frac{q}{2}\right)} \int_{0}^{\infty} \int_{0}^{\infty} \rho_{1}^{p-1} \rho_{2}^{q-1}\left\{\left(1+\rho_{1}^{2}\right)^{2}+\rho_{2}^{2}\right\}^{-\frac{s+\rho_{0}}{2}} \\
& \times \frac{\Gamma(s+1)}{\Gamma(\alpha) \Gamma(\beta)} \sum_{k=0}^{\infty} \frac{\Gamma(\alpha+k) \Gamma(\beta+k)}{\Gamma(s+1+k) k !}\left(\frac{(\cosh t)^{-2}}{\left(1+\rho_{1}^{2}\right)^{2}+\rho_{2}^{2}}\right)^{k} d \rho_{1} d \rho_{2} \\
= & \frac{2 \pi^{\frac{p}{2}}}{\Gamma\left(\frac{p}{2}\right)} \frac{2 \pi^{\frac{q}{2}}}{\Gamma\left(\frac{q}{2}\right)} \frac{\Gamma(s+1)}{\Gamma(\alpha) \Gamma(\beta)} \sum_{k=0}^{\infty} I_{k}(s) \frac{\Gamma(\alpha+k) \Gamma(\beta+k)}{\Gamma(s+1+k) k !}(\cosh t)^{-2 k}
\end{aligned}
$$


with

$$
I_{k}(s)=\int_{0}^{\infty} \int_{0}^{\infty} \rho_{1}^{p-1} \rho_{2}^{q-1}\left\{\left(1+\rho_{1}^{2}\right)^{2}+\rho_{2}^{2}\right\}^{-\frac{s+\rho_{0}}{2}-k} d \rho_{1} d \rho_{2} \quad\left(k \in \mathbf{N}_{0}\right) .
$$

By making the substitution $\rho_{2} \rightarrow\left(1+\rho_{1}^{2}\right) \rho_{2}$, we have the expression for $I_{k}(s)$ as

$$
\int_{0}^{\infty} \rho_{1}^{p-1}\left(1+\rho_{1}^{2}\right)^{-\left(s+\rho_{0}\right)-2 k+q} d \rho_{1} \cdot \int_{0}^{\infty} \rho_{2}^{q-1}\left(1+\rho_{2}^{2}\right)^{-\frac{s+\rho_{0}}{2}-k} d \rho_{2} .
$$

The relation

$$
\int_{0}^{\infty} \rho^{2 x-1}\left(1+\rho^{2}\right)^{-x-y} d \rho=\frac{\Gamma(x) \Gamma(y)}{2 \Gamma(x+y)}, \quad \operatorname{Re}(x), \operatorname{Re}(y)>0
$$

gives

$$
\begin{aligned}
I_{k}(s) & =\frac{1}{2} \frac{\Gamma\left(\frac{p}{2}\right) \Gamma\left(s+\rho_{0}+2 k-q-\frac{p}{2}\right)}{\Gamma\left(s+\rho_{0}+2 k-q\right)} \cdot \frac{1}{2} \frac{\Gamma\left(\frac{q}{2}\right) \Gamma\left(\frac{s+\rho_{0}}{2}+k-\frac{q}{2}\right)}{\Gamma\left(\frac{s+\rho_{0}}{2}+k\right)} \\
& =\frac{1}{4} \Gamma\left(\frac{p}{2}\right) \Gamma\left(\frac{q}{2}\right) \frac{\Gamma(s+2 k) \Gamma\left(\frac{s+\rho_{0}-q}{2}+k\right)}{\Gamma\left(s+\rho_{0}-q+2 k\right) \Gamma\left(\frac{s+\rho_{0}}{2}+k\right)} \\
& =\frac{1}{4} \Gamma\left(\frac{p}{2}\right) \Gamma\left(\frac{q}{2}\right) \frac{\Gamma(s+2 k) \cdot \pi^{\frac{1}{2}} 2^{-\left(s+\rho_{0}-q+2 k-1\right)}}{\Gamma\left(\frac{s+\rho_{0}}{2}+k\right) \Gamma\left(\frac{s+\rho_{0}-q}{2}+k+\frac{1}{2}\right)} \\
& =\frac{1}{4} \Gamma\left(\frac{p}{2}\right) \Gamma\left(\frac{q}{2}\right) \frac{\Gamma(s+2 k) \cdot \pi^{\frac{1}{2}} 2^{-s-\rho_{0}+q+1} \cdot 2^{-2 k}}{\Gamma(\alpha+k) \Gamma(\beta+k)} .
\end{aligned}
$$

The third equality is obtained by the duplication formula:

$$
\Gamma(2 z)=\pi^{-\frac{1}{2}} 2^{2 z-1} \Gamma(z) \Gamma\left(z+\frac{1}{2}\right),
$$

for $2 z=s+\rho_{0}-q+2 k$ and the last one is by $s+\rho_{0}-q+1=s+\frac{p}{2}+1=s+2 m-\rho_{0}=2 \beta$. Substituting (5.12) into (5.9) and using the duplication formula (5.13) for $2 z=s+2 k$, we have

$$
\begin{aligned}
& I(s)=\pi^{\frac{1}{2}(p+q+1)} 2^{-s-\rho_{0}+q+1} \frac{\Gamma(s+1)}{\Gamma(\alpha) \Gamma(\beta)} \sum_{k=0}^{\infty} \frac{\Gamma(s+2 k)}{\Gamma(s+1+k) k !} 2^{-2 k}(\cosh t)^{-2 k} \\
& =\pi^{\frac{1}{2}(p+q)} 2^{-\rho_{0}+q} \frac{\Gamma(s+1)}{\Gamma(\alpha) \Gamma(\beta)} \sum_{k=0}^{\infty} \frac{\Gamma\left(\frac{s}{2}+k\right) \Gamma\left(\frac{s+1}{2}+k\right)}{\Gamma(s+1+k) k !}(\cosh t)^{-2 k} \\
& =2^{-\rho_{0}+q} \pi^{\frac{1}{2}(p+q)} \frac{\Gamma(s+1)}{\Gamma(\alpha) \Gamma(\beta)} \frac{\Gamma\left(\frac{s}{2}\right) \Gamma\left(\frac{s+1}{2}\right)}{\Gamma(s+1)}{ }_{2} F_{1}\left(\frac{s}{2}, \frac{s+1}{2} ; s+1 ; \frac{1}{\cosh ^{2} t}\right) .
\end{aligned}
$$

By $[16,(9.8 .3)$ in p.259]:

$$
{ }_{2} F_{1}\left(\alpha, \alpha+\frac{1}{2} ; 2 \alpha+1 ; z\right)=\left(\frac{1+\sqrt{1-z}}{2}\right)^{-2 \alpha},
$$

we get

$$
I(s)=2^{-\rho_{0}+q} \pi^{\frac{1}{2}(p+q)} \frac{\Gamma\left(\frac{s}{2}\right) \Gamma\left(\frac{s+1}{2}\right)}{\Gamma(\alpha) \Gamma(\beta)} e^{-s t}(2 \cosh t)^{s} .
$$


By (5.6), (5.7) and (5.16), we have

$$
\begin{aligned}
& \int_{N} \phi_{s}^{[0]}(n h) d n \\
= & \frac{c_{G}^{-1}}{(2-2 m)} \int_{\mathfrak{n}_{\beta} \times \mathfrak{n}_{2 \beta}} \phi_{s}^{(2)}\left(\exp u H_{0}\right) d X d Y \\
= & \frac{c_{G}^{-1}}{(2-2 m)} \gamma(s)(\cosh t)^{-s} e^{\rho_{0} t} 2^{\frac{p}{2}} c^{-m+\frac{1}{2}} \cdot I(s) \\
= & \frac{c_{G}^{-1}}{(2-2 m)} \frac{\Gamma(\alpha) \Gamma(\beta)}{\Gamma(s+1) \Gamma(m-1)}(\cosh t)^{-s} \cdot e^{\rho_{0} t} 2^{\frac{p}{2}} c^{-m+\frac{1}{2}} \\
= & \frac{c_{G}^{-1}}{(2-2 m)} \frac{c^{-m+\frac{1}{2}} \pi^{m-\frac{1}{2}}}{\Gamma(m-1)} \frac{2^{s} \Gamma\left(\frac{s}{2}\right) \Gamma\left(\frac{s+1}{2}\right)}{\Gamma(s+1)} e^{-\left(s-\rho_{0}\right) t} \\
= & \frac{c_{G}^{-1}}{(2-2 m)} \frac{2 \pi^{m} c^{-m+\frac{1}{2}}}{\Gamma(m-1)} \frac{e^{-\left(s-\rho_{0}\right) t}}{s} \\
= & \frac{c_{G}^{-1}}{(2-2 m)}(2 m-2) \frac{\pi^{m} c^{-m+\frac{1}{2}}}{\Gamma(m)} \frac{e^{-\left(s-\rho_{0}\right) t}}{s}=-\frac{e^{-\left(s-\rho_{0}\right) t}}{2 s} .
\end{aligned}
$$

The proof for $r=0$ is finished by

$$
\begin{aligned}
& J^{[0]}([\gamma] ; s)=\operatorname{vol}\left(\Gamma_{\gamma} \backslash G_{\gamma}\right) I^{[0]}([\gamma] ; s) \\
= & \operatorname{vol}\left(\Gamma_{\gamma} \backslash G_{\gamma}\right) \operatorname{vol}\left(A \backslash G_{h}\right)^{-1}\left|\operatorname{det}\left(1-\left.\operatorname{Ad}\left(h_{\gamma}\right)\right|_{\mathfrak{n}}\right)\right|^{-1} \int_{N} \phi_{s}^{[0]}(n h) d n \\
= & \operatorname{vol}\left(\Gamma_{\gamma} \backslash G_{\gamma}\right) \operatorname{vol}\left(A \backslash G_{h}\right)^{-1} e^{-2 \rho_{0} t}\left|\operatorname{det}\left(1-\left.\operatorname{Ad}\left(h_{\gamma}^{-1}\right)\right|_{\mathfrak{n}}\right)\right|^{-1} \int_{N} \phi_{s}^{[0]}(n h) d n
\end{aligned}
$$

and [23, Theorem 11.30, p.189] (or [6]),

$$
\operatorname{vol}\left(\Gamma_{\gamma} \backslash G_{\gamma}\right) \operatorname{vol}\left(A \backslash G_{h}\right)^{-1}=t_{\gamma} j(\gamma)^{-1}
$$

Finally we have the formula for $J^{[r]}([\gamma] ; s)(r \geqslant 1)$, by interchanging integration and differentiation.

Recall the integral (4.1), which is expressed by eigenvalues of Laplacian in Proposition 4.2.2. Now we obtain another expression of that integral.

PROPOSITION 5.1.2.

(a) The infinite series

$$
J_{\text {hyp }}(s)=-\sum_{\gamma \in \mathcal{H}(\Gamma)} j(\gamma)^{-1}\left|\operatorname{det}\left(1-\left.\operatorname{Ad}\left(h_{\gamma}^{-1}\right)\right|_{\mathfrak{n}}\right)\right|^{-1} \frac{t_{\gamma} e^{-\left(s+\rho_{0}\right) t_{\gamma}}}{2 s}
$$

converges absolutely and uniformly on $\operatorname{Re}(s) \geqslant \rho_{0}+\epsilon$ for any $\epsilon>0$.

(b) If $r \geqslant m$ and $\operatorname{Re}(s)>\rho_{0}$, then we have

$$
\int_{\Gamma \backslash X} \mathbf{P}_{r+1, s}(\dot{x}, \dot{x}) d \dot{x}=\operatorname{vol}(\Gamma \backslash G)\left(\lim _{g \rightarrow e, g \in G^{+}} \phi_{s}^{[r]}(g)\right)+\sum_{\gamma \in \mathcal{H}(\Gamma)} J^{[r]}([\gamma] ; s),
$$

where the series in the right-hand side of (5.20) converges absolutely and uniformly on $\operatorname{Re}(s) \geqslant \rho_{0}+\epsilon$ for any $\epsilon>0$. 
Proof. The assertion is ensured by the next lemma.

Lemma 5.1.3. Suppose that $\Gamma$ is a discrete subgroup of $G$ such that $\Gamma \backslash G$ is compact. Then the counting function

$$
\pi_{0}(T):=\#\left\{\{\gamma\}_{\Gamma} \in \mathcal{H}(\Gamma) \mid N(\gamma)=e^{t_{\gamma}} \leqslant T\right\}, \quad T>0
$$

satisfies the growth condition

$$
\pi_{0}(T)=O\left(T^{2 \rho_{0}}\right) \text { as } \quad T \rightarrow \infty .
$$

Proof. (Similar to $[4$, Lemma 2.6.3, p.70]) It is well known that $\log N(\gamma)=$ $t_{\gamma}=\inf \{d(x, \gamma x) \mid x \in X\}$. Set $\operatorname{ax}(\gamma)=\left\{x \in X \mid d(x, \gamma x)=t_{\gamma}\right\}$, the axis of $\gamma$. Since $\Gamma \backslash G$ is compact, $\Gamma$ has a compact fundamental domain $X_{\Gamma} \subset X$ such that $\Gamma X_{\Gamma}=X$. Let $\gamma \in \Gamma$, hyperbolic and suppose that $z \in \operatorname{ax}(\gamma)$. Then there is an element $h_{0} \in \Gamma$ such that $h_{0} z \in X_{\Gamma}$ and hence $t_{\gamma}=d(z, \gamma z)=d\left(h_{0} z, h_{0} \gamma h_{0}^{-1} \cdot h_{0} z\right)$ with $h=h_{0} \gamma h_{0}^{-1} \in\{\gamma\}_{\Gamma}$. Let $d(A, B):=\inf \{d(x, y) \mid x \in A, y \in B\}$ denote the hyperbolic distance between the non-empty sets $A, B \subset X$, and $B(x, r)$ the ball with radius $r>0$ centered at $x \in X$. Put $d_{0}:=\sup \left\{d(x, y) \mid x, y \in X_{\Gamma}\right\}$ and choose a point $x_{0} \in X_{\Gamma}$.

$$
\begin{aligned}
\pi_{0}(T) & =\#\left\{\{\gamma\}_{\Gamma} \in \mathcal{H}(\Gamma) \mid N(\gamma)=e^{t_{\gamma}} \leqslant T\right\} \\
& \leqslant \#\left\{h \in \Gamma \mid d\left(X_{\Gamma}, h X_{\Gamma}\right) \leqslant \log T\right\} \\
& \leqslant \#\left\{h \in \Gamma \mid d\left(x_{0}, h x_{0}\right) \leqslant \log T+2 d_{0}\right\} \\
& \leqslant \#\left\{h \in \Gamma \mid h X_{\Gamma} \subset B\left(x_{0}, \log T+3 d_{0}\right)\right\} \\
& \leqslant \frac{\operatorname{vol}\left(B\left(x_{0}, \log T+3 d_{0}\right)\right)}{\operatorname{vol}\left(X_{\Gamma}\right)} \\
& \leqslant \frac{c_{G}}{\operatorname{vol}\left(X_{\Gamma}\right)} \int_{0}^{\log T+3 d_{0}} \mu(t) d t \\
& \leqslant \frac{2^{-(p+2 q)} c_{G}}{\operatorname{vol}\left(X_{\Gamma}\right)} \int_{0}^{\log r-3 d_{0}}\left(e^{t}-e^{-t}\right)^{p+q}\left(e^{t}+e^{-t}\right)^{q} d t \\
& \leqslant \frac{2^{-(p+2 q)} c_{G} e^{(p+2 q) 3 d_{0}}}{(p+2 q) \operatorname{vol}\left(X_{\Gamma}\right)} T^{(p+2 q)} .
\end{aligned}
$$

So we have the desired formula $\pi_{0}(T)=O\left(T^{2 \rho_{0}}\right)$ as $T \rightarrow \infty$.

5.2. The resolvent trace formula. From Proposotion 4.2.2, Proposition 3.1 .5 (iii) and Proposition 5.1.2, we arrive at the formula.

THEOREM 5.2.1. If $r \geqslant m$ and $\operatorname{Re}(s)>\rho_{0}$, then we han,

$$
\sum_{n=0}^{\infty} \frac{1}{\left(s^{2}-s_{n}^{2}\right)^{r+1}}=-\frac{1}{r !}\left(-\frac{1}{2 s} \frac{d}{d s}\right)^{r}\left(J_{\text {id }}(s)+J_{\text {hyp }}(s)\right)
$$

with

$$
\begin{aligned}
J_{\text {id }}(s)= & \operatorname{vol}(\Gamma \backslash G) \frac{2^{-m-3 / 2} \pi^{-m} c_{0}^{m-1 / 2}}{\Gamma(m)}(-1)^{m+1} \\
& \times \prod_{j=1}^{m-1}\left\{\left(\frac{s}{2}\right)^{2}-\left(\frac{\rho_{0}}{2}-j\right)^{2}\right\} \cdot\left\{\psi\left(\frac{s+\rho_{0}}{2}\right)+\psi\left(\frac{s-\rho_{0}}{2}+1\right)\right\} \\
J_{\text {hyp }}(s)=- & \sum_{\gamma \in \mathcal{H}(\Gamma)} j(\gamma)^{-1}\left|\operatorname{det}\left(1-\left.\operatorname{Ad}\left(h_{\gamma}^{-1}\right)\right|_{\mathfrak{n}}\right)\right|^{-1} \frac{t_{\gamma} e^{-\left(s+\rho_{0}\right) t_{\gamma}}}{2 s} .
\end{aligned}
$$




\section{Selberg zeta function.}

6.1. Analytic continuation of the Selberg zeta function. We recall the definition of the Selberg zeta function for $\Gamma \backslash X$ with $\Gamma$ as in the previous section. Let $H$ be a $\theta$-stable Cartan subgroup of $G$ containing $A$. Then $H=A H^{-}$with $H^{-}=H \cap K$. Let $P$ be the set of those $\operatorname{root} \beta$ for $\left(\mathfrak{h}_{\mathbf{C}}, \mathfrak{g}_{\mathbf{C}}\right)$ with $\beta\left(H_{0}\right)>0$, and $\Lambda$ the set of linear forms on $\mathfrak{h}_{\mathbf{C}}$ of the form

$$
\lambda=\sum_{\beta \in P} n_{\beta} \beta, \quad n_{\beta} \in \mathbf{N}_{0} .
$$

For $\lambda \in \Lambda$ let $m_{\lambda}$ denote the number of the ways to express it in the form (6.1).

Let $\operatorname{Prim}(\Gamma)$ be the set of primitive conjugacy classes in $\mathcal{H}(\Gamma)$,i.e., the set of nontrivial $\Gamma$-conjugacy class which is not a power of any other $\Gamma$-conjugacy class. Then for $[\gamma] \in \mathcal{H}(\Gamma)$ there exists a unique $\left[\gamma_{0}\right] \in \operatorname{Prim}(\Gamma)$ such that $[\gamma]=\left[\gamma_{0}^{j(\gamma)}\right]$ with $j(\gamma)$ the multiplicity of $\gamma$.

Since $H^{-}$is a Cartan subgroup of the compact group $M$, any element of $M$ is $M$-conjugate to an element of $H^{-}$. Hence the $G$-conjugacy class of a $[\gamma] \in \mathcal{H}(\Gamma)$ contains an element of $H$ expressed as

$$
h_{\gamma}=\exp \left(t_{\gamma} H_{0}\right) h_{\gamma}^{-}, \quad t_{\gamma}>0, h_{\gamma}^{-} \in H^{-} .
$$

For $\lambda \in \Lambda$ the associated character of $H$ is denoted by $\xi_{\lambda}: H \rightarrow \mathbf{C}^{*}$. With these notations, the Selberg zeta function for $\Gamma \backslash X$ is defined as the Euler product

$$
Z_{\Gamma}(s)=\prod_{[\gamma] \in \operatorname{Prim}(\Gamma)} \prod_{\lambda \in \Lambda}\left(1-\xi_{-\lambda}\left(h_{\gamma}\right) e^{-s t_{\gamma}}\right)^{m_{\lambda}}
$$

It is easy to see that the logarithmic derivative of $Z_{\Gamma}(s)$ is related to the function $J_{\text {hyp }}(s)$ by the formula

$$
-\frac{1}{2 s} \frac{d}{d s} \log Z_{\Gamma}\left(s+\rho_{0}\right)=J_{\text {hyp }}(s)
$$

Hence by Proposition 5.1.2 (a), the infinite product (6.2) converges absolutely and locally uniformly on $\operatorname{Re}(s)>2 \rho_{0}$ defining $Z_{\Gamma}(s)$ holomorphic in $s$ on that half-plane.

Corollary 6.1.1. The Selberg zeta function $Z_{\Gamma}(s)$, defined for $\operatorname{Re}(s)>2 \rho_{0}$, has the analytic continuation as a meromorphic function on the whole complex plane. $Z_{\Gamma}(s)$ has zeros located at $s=\rho_{0} \pm s_{n}, n \geqslant 0$. If $\lambda_{n} \neq \rho_{0}^{2}$, the order of the zeros at $s=\rho_{0} \pm s_{n}$ equals the multiplicity of the eigenvalue $\lambda_{n}$. If $\rho_{0}^{2}$ is an eigenvalue of the Laplacian $\triangle$, then the order of the zero at $s=\rho_{0}$ equals twice the multiplicity of the eigenvalue $\lambda_{k}=\rho_{0}^{2}$.

Proof. By (6.3) and Theorem 5.2.1, we have

$$
\sum_{n=0}^{\infty} \frac{1}{\left(s^{2}-s_{n}^{2}\right)^{r+1}}=-\frac{1}{r !}\left(-\frac{1}{2 s} \frac{d}{d s}\right)^{r}\left(J_{\mathrm{id}}(s)-\frac{1}{2 s} \frac{d}{d s} \log Z_{\Gamma}\left(s+\rho_{0}\right)\right) .
$$

The left-hand side of (6.4) is a meromorphic function of $s \in \mathbf{C}$ and its poles are located at the points $s= \pm s_{n}$ with order $r+1$ (or $2 r+2$ if $s_{n}=0$ ). It is simple to see that $J_{\mathrm{id}}(s)$ is meromorphic on $\mathbf{C}$ and its all the residues are integers. Hence $\frac{d}{d s} \log Z_{\Gamma}(s)$ is a meromorphic function with simple poles only at $s=\rho_{0} \pm s_{n}$ and at 
points coming from $J_{\text {id }}(s)$. For $\lambda_{n} \neq \rho_{0}^{2}$, the residue of $\frac{d}{d s} \log Z_{\Gamma}(s)$ at $s=\rho_{0} \pm s_{n}$ is equal to the multiplicity of $\lambda_{n}$, and if $\rho_{0}^{2}$ is an eigenvalue, the residue of the pole at $s=\rho_{0}$ equals twice the multiplicity of the eigenvalue $\rho_{0}^{2}$. So all the residues at $s=\rho_{0} \pm s_{n}$ are non-negative integers. This completes the proof.

REMARK 1. For almost all $n \geqslant 0, s_{n}$ is purely imaginary.

REMARK 2. We can also show that there exists a meromorphic function $Z_{\text {id }}(s)$ such that

$$
-\frac{1}{2 s} \frac{d}{d s} \log Z_{\mathrm{id}}\left(s+\rho_{0}\right)=J_{\mathrm{id}}(s) .
$$

Since the left-hand side of (6.4) is invariant under $s \rightarrow-s$, the completed Selberg zeta function $\widehat{Z}_{\Gamma}(s):=Z_{\Gamma}(s) Z_{\text {id }}(s)$ satisfy the symmetric functional equation

$$
\widehat{Z}_{\Gamma}\left(2 \rho_{0}-s\right)=\widehat{Z}_{\Gamma}(s) \text {. }
$$

The function $Z_{\text {id }}(s)$ is called gamma factors (or identity factor) of $Z_{\Gamma}(s)$. It is known that $Z_{\text {id }}(s)$ is described by the multiple gamma functions. We refer [15], [21] and [9] for this topic.

\section{REFERENCES}

[1] ArakaWA, T., Selberg zeta functions associated with a theta multiplier system of $S L_{2}(\mathbf{Z})$ and Jacobi forms, Math. Ann., 293 (1992), pp. 213-237.

[2] Bruggeman, R.W., Miatello, R.J., Wallach, N.R., Resolvent and lattice points on symmetric spaces of strictly negative curvature, Math. Ann., 315 (1999), pp. 617-639.

[3] Elstrodt, J., Die Resolvente zum Eigenwertproblem der automorphen Formen in der hyperbolischen Ebene, Teil I, Math. Ann., 203 (1973), pp. 295-330.

[4] Elstrodt, J., Grunewald, F., And Mennicke, J., Groups Acting on Hyperbolic Space: Harmonic Analysis and Number Theory, Springer-Verlag, 1998.

[5] Fischer, J., An approach to the Selberg trace formula via the Selberg zeta-function, Lecture Notes in Math., 1253 (1987), Springer-Verlag.

[6] Gangolli, R., The length spectra of some compact manifolds of negative culvature, J. Diff. Geom., 12 (1977), pp. 403-424.

[7] Gangolli, R., Zeta functions of Selberg's type for compact space forms of locally symmetric spaces of rank one, Illinoi J. Math., 21 (1977), pp. 1-41.

[8] Gangolli, R., Warner, G., Zeta functions of Selberg's type for some noncompact quotients of symmetric space of rank one, Nagoya Math. J., 78 (1980), pp. 1-44.

[9] GoN, Y., Gamma factors of Selberg zeta functions and functional equation of Ruelle zeta functions, Math. Ann., 308, no. 2, (1997), pp. 251-278.

[10] Hejahl, D.A., The Selberg trace formula for PSL(2, R) II, Lecture Notes in Math., 1001 (1983), Springer Verlag, Berlin.

[11] Helgason, S., Differential Geometry, Lie Groups, and Symmmetric Spaces, Academic Press, 1978.

[12] Helgason, S., Groups and Geometric Analysis, Academic Press, 1984.

[13] JuHL, A., Cohomological Theory of Dynamical Zeta Functions, Birkhäuser, 2001.

[14] KNAPP, A.W., Representation theory of semisimple groups, Princeton University Press, 1986.

[15] Kurokawa, N., Gamma factors and Plancherel measures, Proc. Japan Acad. Ser A., 68 (1992), pp. 256-260.

[16] Lebedev, N. N., Special functions and their applications, Dover Publications, INC., New York 1972.

[17] Miatello, R.J., Wallach, N.R., The resolvent of the Laplacian on locally symmetric spaces, J. Diff. Geom., 36, pp. 663-698.

[18] Oda, T., Tsuzuki, M., Automorphic Green functions associated with the secondary spherical functions, preprint, UTMS 2000-34 (2000).

[19] RoElcke, W., Das Eigenwertproblem der automorphen Formen in der hyperbolishen Ebene I, II, Math. Ann., 167 (1996), pp. 292-337 and ibid., 168 (1967), pp. 261-324. 
[20] Selberg, A., Harmonic analysis and discontinuous groups in weakly symmetric Riemannian spaces with applications to Dirichlet series, J. Indian. Soc., (N.S.)(1956), pp. 47-87.

[21] TsuzUKI, M., Elliptic factors of Selberg Zeta functions, Duke Math. J., 88, no. 1, (1997), pp. 29-75.

[22] Whittaker, E. T., Watson, G. N., A course of modern analysis, Cambridge University Press (1963).

[23] Williams, F. L., Lectures on the spectrum of $L^{2}(\Gamma \backslash G)$, Pitman Research Notes in Math., 242 (1991). 